\title{
$R$ as an Environment for Reproducible Analysis of DNA Amplification Experiments
}

by Stefan Rödiger, Michał Burdukiewicz, Konstantin Blagodatskikh, Michael Jahn and Peter Schierack

\begin{abstract}
There is an ever-increasing number of applications, which use quantitative PCR (qPCR) or digital PCR (APCR) to elicit fundamentals of biological processes. Moreover, quantitative isothermal amplification (qIA) methods have become more prominent in life sciences and point-of-carediagnostics. Additionally, the analysis of melting data is essential during many experiments. Several software packages have been developed for the analysis of such datasets. In most cases, the software is either distributed as closed source software or as monolithic block with little freedom to perform highly customized analysis procedures. We argue, among others, that $\mathrm{R}$ is an excellent foundation for reproducible and transparent data analysis in a highly customizable cross-platform environment. However, for novices it is often challenging to master $\mathrm{R}$ or learn capabilities of the vast number of packages available. In the paper, we describe exemplary workflows for the analysis of qPCR, qIA or dPCR experiments including the analysis of melting curve data. Our analysis relies entirely on $\mathrm{R}$ packages available from public repositories. Additionally, we provide information related to standardized and reproducible research.
\end{abstract}

\section{Introduction}

Quantitative polymerase chain reaction (qPCR) is the method of choice when a precise quantification of minute DNA traces is required. Applications include the detection and quantification of pathogens or gene expression analysis (Pabinger et al., 2014). Only few bioanalytical methods had such a significant impact on the progress of life sciences and medical sciences as the qPCR (Huggett et al., 2015b). Numerous commercial and experimental monitoring platforms have been developed in the past years. This includes standard plate cyclers, capillary cyclers, microfluidic platforms and related technologies (Rödiger et al., 2013b; Viturro et al., 2014; Rödiger et al., 2014; Khodakov and Ellis, 2014; Wu et al., 2014).

In the past decades several isothermal amplification technologies emerged, such as helicase dependent amplification (HDA). Isothermal amplification methods were readily combined with realtime monitoring technologies (qIA) or digital PCR and are used in various fields like diagnostics and point-of-care-testing (Selck et al., 2013; Rödiger et al., 2014; Nixon et al., 2014).

Digital PCR (dPCR) is a novel approach for detection and quantification of nucleic acids and can be seen as a next generation method (Huggett et al., 2015b). The dPCR technology breaks fundamentally with the previous concept of nucleic acid quantification. In contrast to traditional qPCR measures dPCR absolute nucleic acids amounts. This is possible after 'clonal DNA amplification' in thousands of small separated partitions (e.g., droplets, nano chambers; Huggett et al. 2013; Milbury et al. 2014; Morley 2014). Partitions with no nucleic acid remain negative and the others turn positive (e.g., Figure 1). Selected technologies (e.g., OpenArray®real-time PCR system) monitor amplification reactions in the chambers ('partitions') in real-time. After that, all quantification cycle (Cq) values are calculated from the amplification curves and converted into discrete events of positive and negative chambers. Finally, the absolute quantification of nucleic acids is done using Poisson statistics (Milbury et al., 2014; Morley, 2014). A representative workflow using the vendor software for the analysis of a droplet dPCR experiment has been described by Milbury et al. (2014). Recently, we have published the dpcR package (Burdukiewicz et al., 2015) at CRAN, which is the first open source R software package for the analysis of $\mathrm{dPCR}$ experiments (see the $\mathbf{d p c R}$ package manual for details).

The complexity of hardware, wetware and software requires expertise to master a workflow. This comprises standards for experiment design, generation and analysis of data, multiple hypothesis testing, interpretation, reporting and storage of results (Huggett et al., 2014; Castro-Conde and de Uña-Álvarez, 2014). Scientific misconduct and fraud have shaken the scientific community on several occasions (Bustin, 2014). In particular, the scientific community works hard to uncover pitfalls of qPCR experiments. This led to the development of peer-reviewed quantification cycle (Cq) analysis algorithms (Ruijter et al., 2013), fully characterized qPCR chemistries (Ruijter et al., 2014) and guidelines for a proper conduct of qPCR experiments as implemented in the MIQE guidelines (minimum information for publication of quantitative real-time PCR experiments; Huggett et al. 2013; Bustin 2014). We share the philosophy of the MIQE guidelines to increase the experimental transparency for better experimental practice and reliable interpretation of results and encourage the 


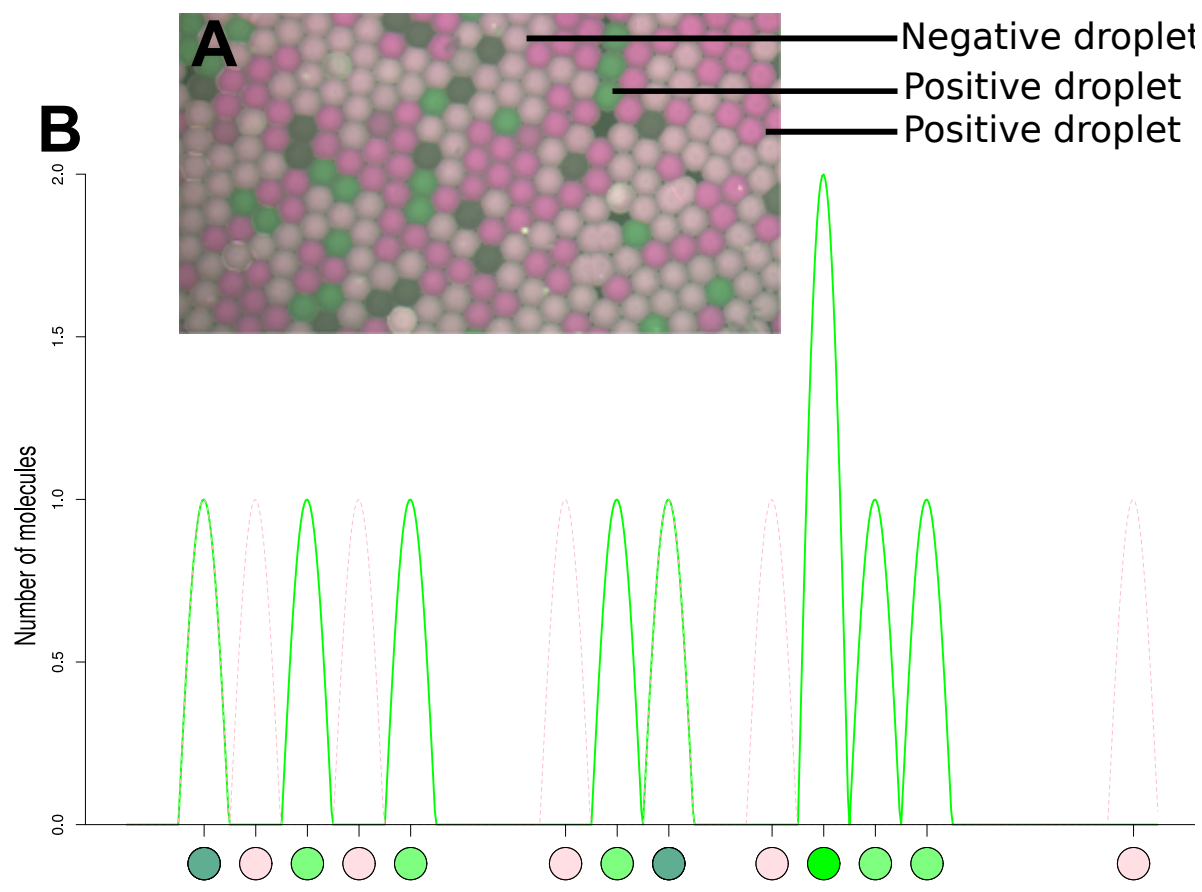

Figure 1: Scheme of a digital PCR experiment. (A) A droplet digital PCR reaction mix was formed in a BioRad QX100 Droplet Digital PCR System. Droplets (circa $100 \mu \mathrm{m}$ in diameter) were subjected to custom made slide chambers for the detection and analysis in fully automatized imaging VideoScan platform (Rödiger et al., 2013b). (B) Subsequently the samples can be digitalized by counting the number of positive and total number of droplets. The plot was generated with the sim_ddpcr function from the dpcR package.

use of open data exchange formats like the XML-based real-time PCR data markup language (RDML; Lefever et al. 2009).

In case of closed source software the analysis usually happens in a black box fashion tied to a specific platform. We agree that closed frameworks are not necessarily a bad thing, but should be avoided if possible (Rödiger et al., 2013a; Spiess et al., 2015). Studies by McCullough and Heiser (2008); Almiron et al. (2010); Durán et al. (2014) exemplified where the black box approach might fail in science. The same holds true for the open source software since software bugs are independent of a development model. However, at least open source gives the possibility to track and eliminate errors by an individual entity. Black-box systems often force the user to process the data by suboptimal analysis algorithms (Ruijter et al., 2013). Moreover, the data usually cannot be accessed between the steps of the analysis, which restrains quality control. Visualization options are usually limited by the software vendor preferences and do not attain publication quality. In addition to this, users who have no access to the commercial software are barred. Aside from closed source software, data analysis is often performed in spreadsheets. However, this data processing approach is not advisable for research purposes. Most spreadsheets lack (or do not use) tools to validate the input, to debug implemented procedures and to automatize the workflow. These traits make them prone to errors and not well suited for complicated tasks (McCullough and Heiser, 2008; Burns, 2014).

For several reasons, $\mathrm{R}$ is one of the most popular tools in bioinformatics and is known as an early adopter of emerging technologies (Pabinger et al., 2014). R provides packages to build highly customized workflows, covering: data read-in, data pre-processing, analysis, post-processing, visualization (Murrell, 2012, 2015) and storage. As recently briefly reviewed in Pabinger et al. (2014), numerous R packages have been developed for the analysis of qPCR, dPCR, qIA and melting curve analysis experiments, including: kulife (Ekstrom et al., 2013), MCMC.qpcr (Matz, 2015), qPCR.CT (Pan et al., 2012), DivMelt (Swan, 2013), qpcR (Spiess, 2014), dpcR, chipPCR (Roediger and Burdukiewicz, 2014), MBmca (Roediger, 2015), RDML (Blagodatskikh et al., 2015), nondetects (McCall1 et al., 2014), qpcrNorm (Mar, 2009), HTqPCR (Dvinge and Bertone, 2009), SLqPCR (Kohl, 2007), ddCt (Zhang et al., 2015), EasyqpcR (Pape, 2012), unifiedWMWqPCR (Neve et al., 2014; Neve and Meys, 2014), ReadqPCR and NormqPCR (Perkins et al., 2012) All packages are either available from CRAN or Bioconductor (Gentleman et al., 2004) and can be freely combined in a plugin-like architecture.

$\mathrm{R}$ is an open, operating system-independent platform for a broad spectrum of calculation options. Particularly, the visualization of experiments is one of R's pinnacles. R enables the users to create an 


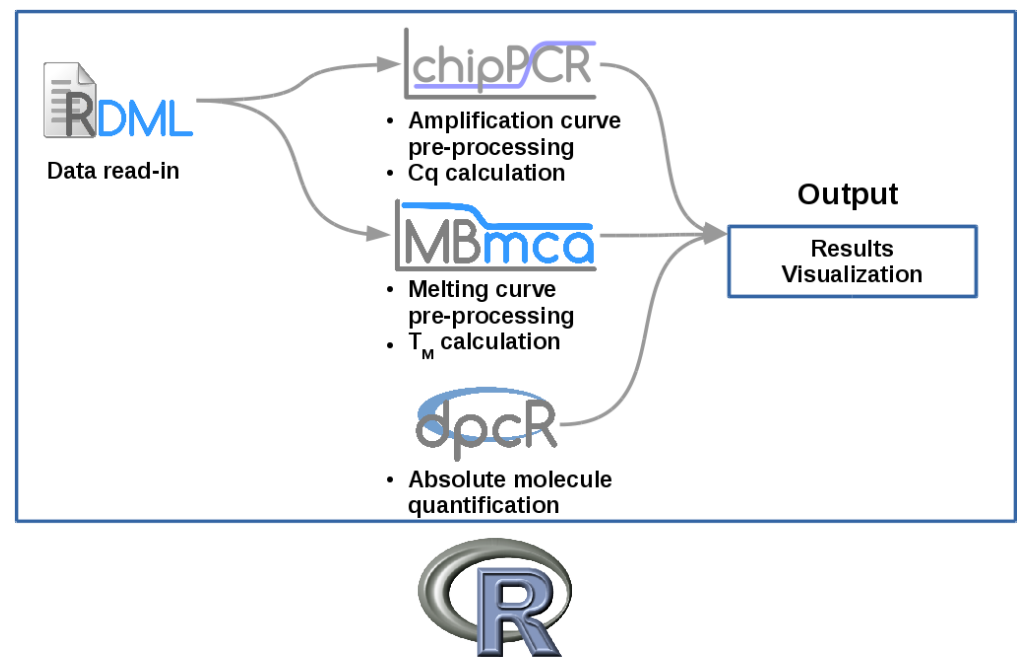

Figure 2: An exemplary workflow for quantitative PCR, digital PCR, quantitative isothermal amplification and melting curve analysis experiments in $\mathrm{R}$. The $\mathrm{R}$ software environment provides core functionality for statistical computing and graphics. In our scenario we used the RDML package to read-in data in standardized format. However, any format supported by $\mathrm{R}$ can be used. Further, processing of amplification curve data was performed with the chipPCR package and melting curve data were analysed with the MBmca package. The dpcR package can be embedded in the analysis of digital PCR experiments. Cq, quantification cycle; $T_{M}$, melting temperature.

efficient manipulation, restructuring and reshaping of data to make them readily-available for further processing. This is of the particular importance to the human-machine interface (Oh, 2014). Intrinsic properties of R such as naming conventions (Bååth, 2012) and class systems (e.g., S3, S4, reference classes and R6) vary considerable, depending on the package developer preferences. However, due to the open source approach, there is the common ground to track numerical errors. R offers various methods for a standardized data import/export and exchange. Workflows can embed structured models (Zeller et al., 2009), open data exchange formats (e.g., RDML), binary formats (Michna and Woods, 2013) or tools provided by the R workspace (R Core Team, 2015). The NetCDF binary format, available from the RNetCDF package (Michna, 2015), has advantages over some other binary formats (e.g., the RData format), since arbitrary array data sections of massive datasets can be processed efficiently (Michna and Woods, 2013). This might be useful for large data sets as present in highthroughput PCR experiments or APCR experiments with large partition numbers. The R environment offers several datasets, which can be used for testing of algorithms. Therefore, we, among others, argue that R is suitable for reproducible research (Gentleman et al., 2004; Murrell, 2012; Gandrud, 2013; Hofmann et al., 2013; Ooms, 2013; Kuhn, 2015; Leeper, 2014; Liu and Pounds, 2014).

The aim of this paper is to show case studies for qPCR, dPCR, qIA and melting curve analysis experiments. Our workflow effectively follows the principle illustrated in Figure 2. We intend to aggregate functionalities dispersed between various packages and offer a fast insight in the analysis of nucleic acid experiments with R. In particular, we describe how to:

- read-in data from a standardized file format,

- pre-process the amplification curve data,

- calculate specific parameters from the amplification curve data,

- calculate the melting temperature,

- and report the data.

\section{Setting-up a working environment}

We recommend performing the scripting in a dedicated integrated development environment (IDE) and graphical user interface (GUI) such as RKWard (Rödiger et al., 2012), RStudio (RStudio, 2014; Gandrud, 2013) or other technologies (Valero-Mora and Ledesma, 2012). Benefits of IDEs with GUI include syntax-highlighting, auto completion and function references for rapid prototyping of workflows. Typically, the analysis will start with data from a commercial platform. Most platforms have an option to export a CSV file or spreadsheets application file (e.g., *.xls, *.odt). Details for the data import have been described elsewhere (R Core Team, 2015; Rödiger et al., 2012). To keep the 
case study sections compact we have chosen to load datasets from the $\mathbf{q p c R}$ package (Ritz and Spiess, 2008) (v. 1.4.0) and the RDML package (v. 0.8-3) to our workspace. The chipPCR package (Rödiger et al., in press) (v. 0.0.8-10) was used for data pre-processing, quality control and the calculation of the quantification cycle $(\mathrm{Cq})$.

The $\mathrm{Cq}$ is a quantitative measure, which represents the number of cycles needed to reach a user defined threshold signal level, in the exponential phase of a qPCR/qIA reaction. Several Cq methods have been described (Ruijter et al., 2013). In this study we have chosen the second derivative maximum method $\left(C q_{S D M}\right)$ and the 'Cycle threshold' $\left(C q_{C t}\right)$ method.

During a perfect qPCR reaction, the target DNA doubles $\left(2^{n} ; n=\right.$ cycle number $)$ at each cycle. Here the amplification efficiency $(A E)$ is $100 \%$. However, in reality, numerous factors cause an inhibition of the amplification $(A E<100 \%)$. The $A E$ can be determined by the relation of the $\mathrm{Cq}$ value depending on the sample input quantity as described in (Rödiger et al., in press; Svec et al., 2015).

In Rödiger et al. (2013a) we described the application of $\mathrm{R}$ for the analysis of melting curve experiments from microbead-based assays. We used functions from the MBmca package (0.0.3-4) for an analysis of the target specific melting temperature $\left(T_{M}\right)$ in experiments of the present study, since the mathematical foundation is the same.

We completed our examples with case studies for the analysis of APCR experiments. In particular, we used the dpcR package (v. 0.1.4.0) to estimate the number of molecules in a sample.

\section{Results}

In the following sections we will show how $\mathrm{R}$ can be used (I) as a unified open software for data analysis and presentation in research, (II) as software frame-work for novel technical developments, (III) as platform for teaching of new technologies and (IV) as reference for statistical methods.

\section{Case study one - qPCR and amplification efficiency calculation}

The goal of our first case study was to calculate the Cq values and the $A E$ from a qPCR experiment. We used the guescini 1 dataset from the qpcR package, where the gene NADH dehydrogenase 1 (MT-ND1) was amplified in a LightCyclerß 480 (Roche) thermocycler. Details of the experiment are described in Guescini et al. (2008). We started with loading the required packages and datasets. A good practice for reproducible research is to track the package versions and environment used during the analysis. The function sessionInfo from the utils package provides this functionality. Assuming that the analysis starts with a clean R session it is possible to assign the required packages to an object, as shown below. Reproducibility of research can be further improved by the archivist package (Biecek and Kosinski, 2015), which stores and recovers crucial data and preserves metadata of saved objects (not shown). All settings of an R session can be easily saved and/or restored using the settings package (van der Loo, 2015).

\# Load the required packages for the data import and analysis.

\# Load the chipPCR package for the pre-processing and curve data quality

\# analysis and load the qpcR package as data resource.

require(chipPCR)

require (qpcR)

\# Collect information about the $\mathrm{R}$ session used for the analysis of the

\# experiment.

current.session <- sessionInfo()

\# Next, we load the 'guescini1' dataset from the qpcR package to the

\# workspace and assign it to the object 'gue'.

gue $<-$ guescini 1

\# Define the dilution of the sample DNA quantity for the calibration curve \# and assign it to the object 'dil'.

dil $<-10^{\wedge}(2:-4)$

We previewed the amplification curve raw data using the matplot function (see code below). The amplification curve data showed a strong signal level variation in the plateau region (Figure 3A). Therefore, all data were subjected to a minimum-maximum normalization using the CPP function from the chipPCR package. In addition, all data were baselined and smoothed (Figure 3B; see Rödiger 
et al. 2013a; Rödiger et al. in press). The Cq values were calculated by the $C q_{S D M}$ and $C q_{C t}$ methods as shown next.

\# Pre-process the amplification curve data with the CPP function from the

\# chipPCR package. The trans parameter was set TRUE to perform a baselining and

\# the method.norm parameter was set to minm for a min-maximum normalization. All

\# amplification curves were smoothed by Savitzky-Golay smoothing.

res.CPP <- cbind(gue[, 1], apply(gue[, -1], 2, function(x) \{

$\operatorname{CPP}($ gue $[, 1], x$, trans $=$ TRUE, method.norm $=$ "minm", method.reg = "least",

\}))

bg. range $=c(1,7))[[" y \cdot$ norm"] $]$

\# Use the th.cyc function from the chipPCR package to calculate the Cq values

\# by the cycle threshold method at a threshold signal level " $r$ " of 0.05 .

Cq.Ct <- apply(gue[, -1], 2, function(x)

th. $\operatorname{cyc}($ res.CPP $[, 1], x, r=0.05)[1])$

\# Use the inder function from the chipPCR package to calculate the $\mathrm{Cq}$ values

\# by the SDM method. This will give a lot of output in the console.

Cq.SDM <- apply(gue[, -1], 2, function $(x)$

summary (inder (res.CPP $[, 1], x))[2]$ )

\# Fit a linear model to carry out a regression analysis.

res. Cq $<-\operatorname{lm}(\mathrm{Cq} . \mathrm{Ct} \sim \mathrm{Cq} . \mathrm{SDM})$

To compare the $C q_{S D M}$ and $C q_{C t}$ methods we performed a regression analysis. The $C q$ methods are in a good agreement. However, the dispersion of the $C_{C_{C}}$ values appeared to be higher than in the $C q_{S D M}$ values (Figure $3 \mathrm{C}$ ).

summary (res.Cq)

Call:

$\operatorname{lm}($ formula $=$ Cq.Ct $\sim$ Cq. SDM $)$

Residuals:

Min $1 Q$ Median $3 Q \quad$ Max

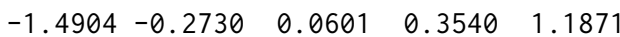

Coefficients:

Estimate Std. Error $t$ value $\operatorname{Pr}(>|t|)$

(Intercept) $-8.125534 \quad 0.207419 \quad-39.17 \quad<2 \mathrm{e}-16 * * *$

Cq.SDM $\quad 0.988504 \quad 0.008097 \quad 122.08 \quad<2 \mathrm{e}-16 * * *$

Signif. codes: 0 '***' 0.001 '**' $0.011^{\prime} * x^{\prime} 0.05$ '.' 0.1 ' ' 1

Residual standard error: 0.5281 on 82 degrees of freedom

Multiple R-squared: 0.9945 , Adjusted R-squared: 0.9945

F-statistic: $1.49 \mathrm{e}+04$ on 1 and $82 \mathrm{DF}, \mathrm{p}$-value: $<2.2 \mathrm{e}-16$

The dilution ('dil') and the $\mathrm{Cq}$ ('Cq.Ct') values served as input for the calculation of the amplification efficiency $(A E)$ with effcalc function from the chipPCR package. In our case study we needed to rearrange the ' $\mathrm{Cq}$. $\mathrm{Ct}^{\prime}$ 'values in a matrix using the command effcalc(dil, $\mathrm{t}$ (matrix(Cq. Ct, nrow $=12, \mathrm{ncol}=7)$ ). For visualization of the confidence intervals of the regression analysis we set the parameter CI = TRUE.

\# Arrange and plot the results in a convenient way.

layout (matrix $(\mathrm{c}(1,2,3,3,4,5), 3,2$, byrow = TRUE $))$

\# Store used margin parameters

def.mar <- par ("mar")

layout (matrix (c (1, 2, 3, 3, 4, 5), 3, 2, byrow = TRUE))

\# Set bigger top margin.

$\operatorname{par}(\operatorname{mar}=c(5.1,4.1,6.1,2.1))$ 

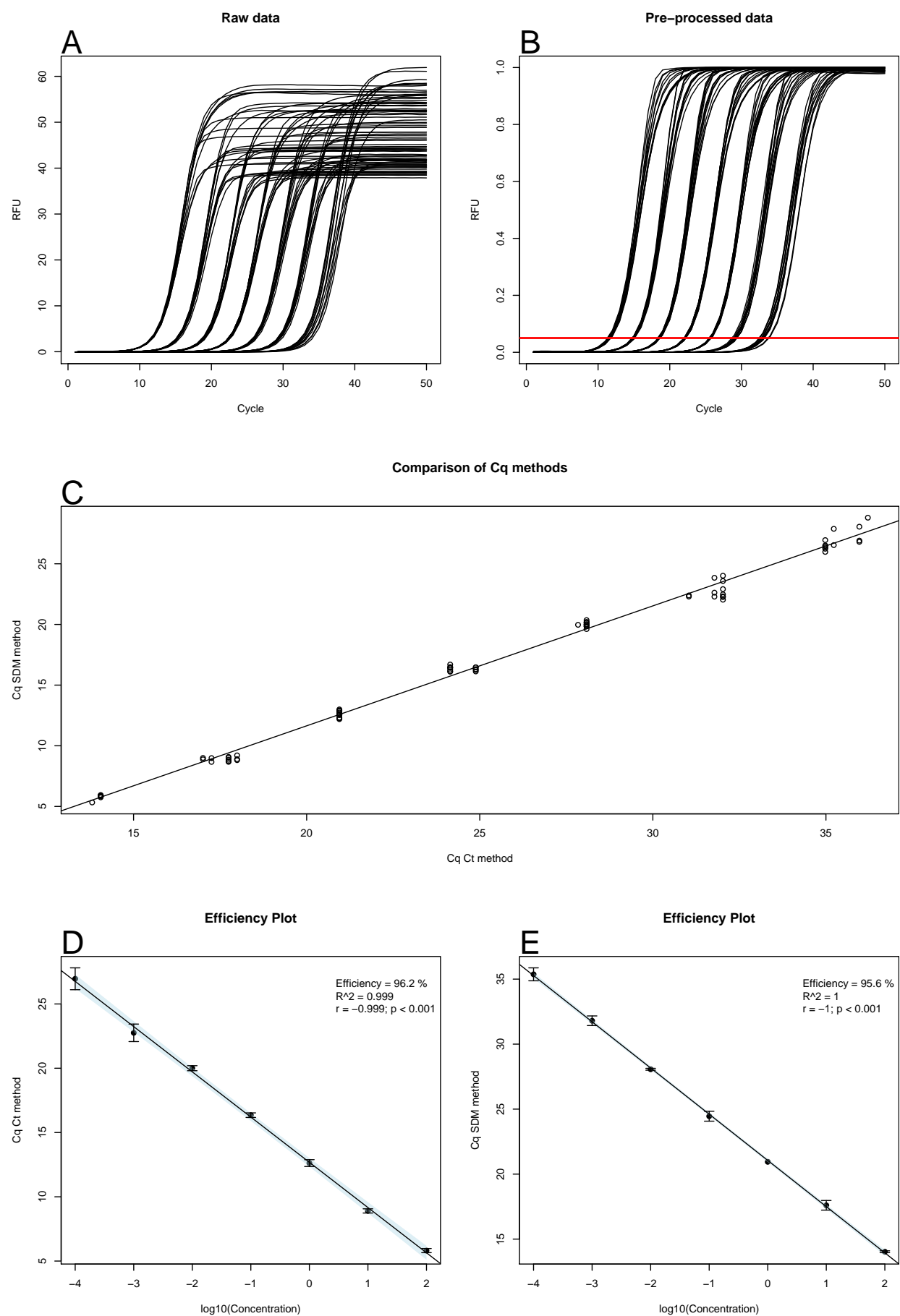

Figure 3: Analysis of the amplification curve data of the guescini 1 dataset. (A) Raw data from the calibration curve samples were visually inspected. The qPCR curves display a broad variation in plateau fluorescence (38-62 RFU). (B) The CPP function from the chipPCR package was used to baseline the data, to smooth the data with Savitzky-Golay smoothing filter and to normalize the data between 0 and 1 . The red horizontal line (-) indicates the fluorescence level (0.05) used for the calculation of the Cq value by the 'cycle threshold' method. (C) The Cq values were calculated by the C $q_{S D M}$ method ('SDM method') (inder, chipPCR) and the $C q_{C t}$ method ('Ct method') (th. cyc, chipPCR). The threshold value was set to $r=0.05$. The $C q_{S D M}$ and $C q_{C t}$ values were plotted and analysed by a linear regression $\left(R^{2}=0.9945 ; P<2.2^{-16}\right)$ and Pearson's $r\left(r=0.9972605 ; P<2.2^{-16}\right)$. The amplification efficiency based on (D) $C q_{C t}$ values and (E) $C q_{S D M}$ values were automatically analysed with the effcalc (chipPCR) function. Cq, Quantification cycle; SDM, Second derivative maximum, $\mathrm{R}^{\wedge} 2$, Coefficient of determination; $\mathrm{r}$, Pearson product-moment correlation coefficient, $\mathrm{RFU}$, relative fluorescence units. 


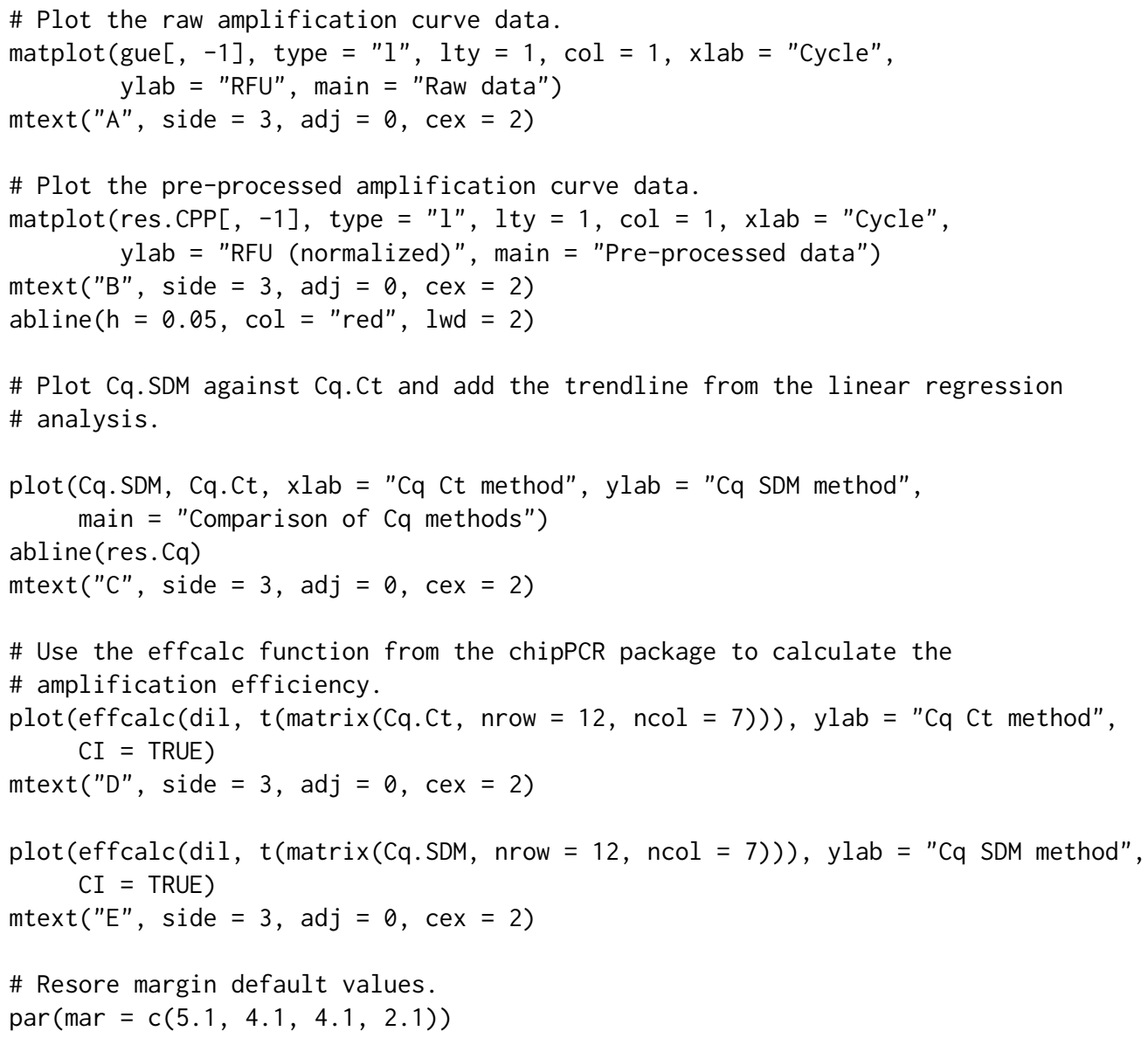

Finally, Cq values were plotted using the layout function (Figures $3 \mathrm{D}$ and $\mathrm{E}$ ). The Cq values and amplification vary slightly between both methods. This is an expected observation and is in accordance to the findings by Ruijter et al. (2013). As shown in this case study, it is easy to set-up a streamlined workflow for data read-in, pre-processing and analysis with a few functions.

\section{Case study two - qPCR and melting curve analysis}

A common task during the analysis of qPCR experiments is to distinguish between positive and negative samples (see Figure 5). If the melting temperature of a sample is known it is possible to automatize the decision by a melting curve analysis (MCA). As shown in Rödiger et al. (2013a) this can be done by interrogating the $T_{M}$. Therefore, we used a logical statement, which tests if $T_{M}$ is within a tight temperature range. We used the signal height as second parameter. In line with 'Case study one qPCR and amplification efficiency calculation we used the function sessionInfo to track all packages used during the analysis. Reproducible research is greatly enhanced if open data exchange formats are used. Therefore, we used the RDML package for data read-in. The amplification and melting curve data were measured with a CFX96 system (Bio-Rad) and then exported as RDML v 1.1 format file as 'BioRad_qPCR_melt.rdml'. Within this qPCR experiment we amplified the Mycobacterium tuberculosis $k a t G$ gene and tried to detect a mutation at codon 315 . The experiment was separated in two parts:

1. Detection of overall M. tuberculosis DNA (wild-type and mutant) and

2. specific detection of wild-type M. tuberculosis by melting of TaqMan probe (quencher - BHQ2, fluorescent reporter - Cy5) with amplified DNA (see Luo et al. 2011 for probe/primer sequences and further details).

The qPCR was conducted using EvaGreen $($ ) Master Mix (Syntol) according to the manufacturer's instructions, with $500 \mathrm{nM}$ of primers and probe in a $25 \mu \mathrm{L}$ final reaction volume. Thermocycling was conducted using a CFX96 (BioRad) initiated by $3 \mathrm{~min}$ incubation at $95^{\circ} \mathrm{C}$, followed by $41 \mathrm{cycles}(15 \mathrm{~s}$ at $95^{\circ} \mathrm{C} ; 40 \mathrm{~s}$ at $65^{\circ} \mathrm{C}$ ) with a single read-out taken at the end of each cycle. Probe melting was conducted between $35^{\circ} \mathrm{C}$ and $95^{\circ} \mathrm{C}$ by $1^{\circ} \mathrm{C}$ at 1 s steps.

RDML file structures can be complex. Though RDML files are XML structured files and thus intended to be readable by humans, it is hard to grasp the complex hierarchical file structure without some basic understanding. A simple and fast method to compactly display the structure of an object 
in $\mathrm{R}$ is to use the str or summary function (not shown). However, such $\mathrm{R}$ tools are not informative in this context. Therefore we implemented a dendrogram-like view (Figure 4). According to this, the file contains different datasets, each with 3 samples, ('pos', 'ntc', 'unknown'). Only a subset of the data was used in our case study and combined to the object qPCR.

\# Import the qPCR and melting curve data via the RDML package.

\# Load the chipPCR package for the pre-processing and curve data quality

\# analysis and the MBmca package for the melting curve analysis.

require ( $R D M L)$

require(chipPCR)

require(MBmca)

require(dplyr)

\# Collect information about the $\mathrm{R}$ session used for the analysis of the GPCR

\# experiment.

current.session <- sessionInfo()

\# Load the BioRad_qPCR_melt.rdml file from the RDML package and assign the data

\# to the object 'BioRad'.

filename <- file.path (path.package("RDML"), "extdata", "BioRad_qPCR_melt.rdml")

BioRad <- RDML\$new(filename)

\# The structure of the file can be overviewed by the AsDendrogram() function.

\# We can see that our experiment contains two detection channels (Figure 4).

\# ('EvaGreen' and 'Cy5' at 'Run ID'). 'EvaGreen' channel has one

\# probe (target) - 'EvaGreen'. 'Cy5' has: 'Cy5', 'Cy5-2' and 'Cy5-2_rr'.

\# each target has three sample types (positive, unknown, negative).

\# And each sample type has qPCR ('adp') and melting ('mdp') data.

\# The last column shows the number of samples in an experiment.

BioRad $\$$ AsDendrogram( $)$

\# Fetch cycle dependent fluorescence for the EvaGreen channel and row 'D'

\# (contains the target 'Cy5-2' in the channel 'Cy5') of the

\# katG gene and aggregate the data in the object 'qPCR'.

qPCR $<-$ BioRad\$AsTable() \% \%

filter(target == "EvaGreen", grepl("^D", position)) \%>\% BioRad\$GetFData(.)

We inspected and pre-processed a subset of the amplification curve data solely using functionalities provided by the chipPCR package. The plotCurves function was used to get an overview of the curvatures. The green background colour of subplots shows that the data contain no missing values. Some curves indicated a moderate baseline shift with a slight negative trend (Figure 5). This observation suggested to baseline the raw data by using a linear regression model (cycles $\mathrm{x}-\mathrm{y}$; $($ bg.range $=c(x, y)$ ) in the CPP function.). The curvatures of 'D1_Alm12...' and 'D2_Alm12...' exhibited a drop in the plateau phase. However, this is not of importance during this stage of the analysis.

\# Use plotCurves function to get an overview of the amplification curve samples \# (Figure 5).

plotCurves(qPCR $[, 1]$, qPCR $[,-1]$, type $=" 1 ")$

mtext ("Cycles", SOUTH <- 1, line = 3)

mtext("Fluorescence", side $=2$, line $=2$ )

Since the amplification curves indicated that selected samples (except non-template-control; 'NTC') are positive, we distinguished between true positive and true negative samples by MCA (Figure 6A).

\# To detect positive samples we calculated the Cq values by the cycle threshold

\# method. This method is implemented in the th.cyc function. The threshold signal

\# level $r$ was set to 10 .

Cq.Positive <- t(apply(qPCR $[,-1], 2$, function $(x)$

\{

res <- $\operatorname{CPP}(\mathrm{qPCR}[, 1], \mathrm{x}$, trans $=$ TRUE, bg. range $=\mathrm{c}(2,8)$, method.reg $=$ "least") $[[" y$. norm" $]]$ 


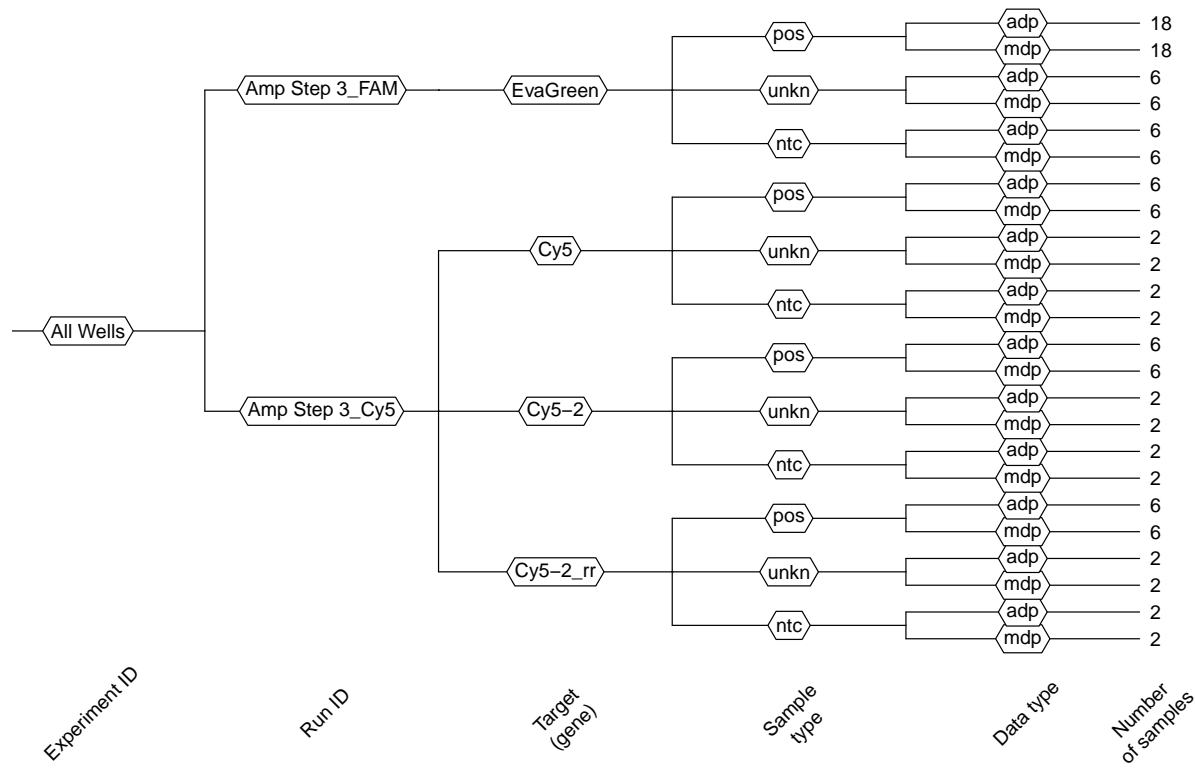

Figure 4: File structure visualization of the RDML file 'BioRad_qPCR_meltrdml' form the RDML package. The file was read by the RDML function and the structure displayed as dendrogram by the call BioRad\$AsDendrogram(). Names are used according to the RDML convention by Lefever et al. (2009). The object BioRad branches into an experiment with Run ID names for two fluorescence detection channels (FAM, Cy5). The targets have typical designations like pos (positive), unkn (unknown) and ntc (non-template control). In the deeper branches are the data types adp (amplification data point) and mdp (melting data point) shown with the number of samples (ranging from 2 to 18).

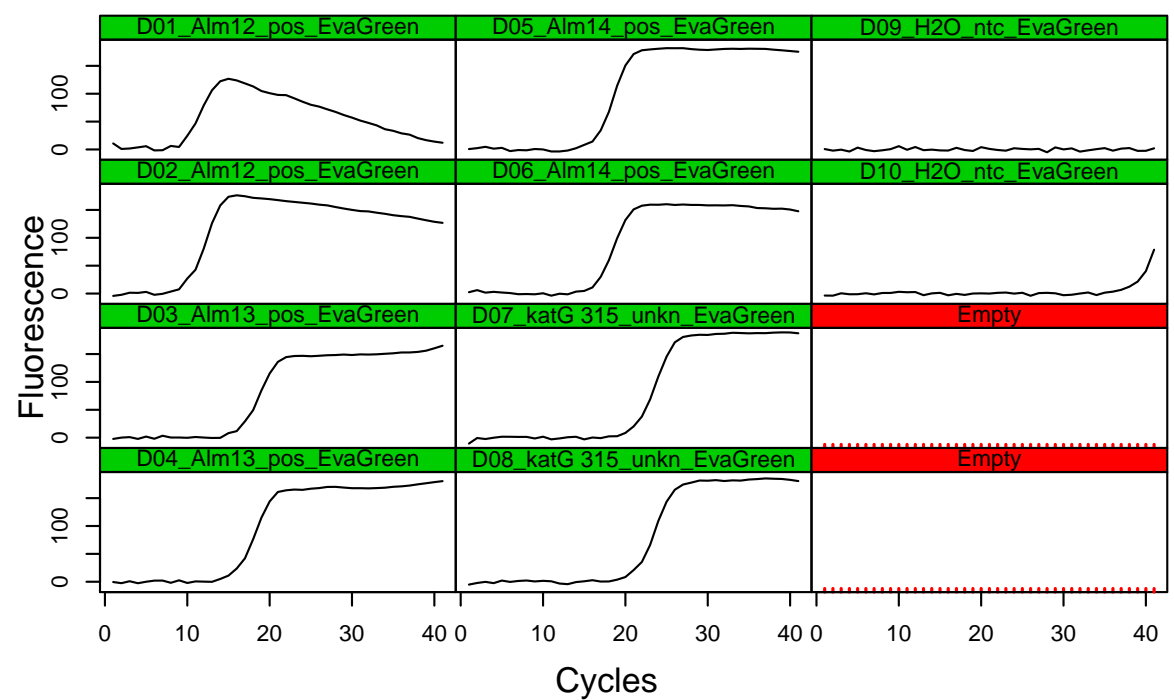

Figure 5: Analysis of the amplification curve data. The calibration curve samples were inspected by the plotCurves function from the chipPCR package. The green colour code indicates that the data contain no missing values. However, the visual inspection revealed that the data are noisy. All samples ('D1_Alm12...'-D8_Alm12...') appeared to be positive. One negative control ('D10_H20_ntc_EvaGreen') seems to be contaminated.

\# The th.cyc fails when the threshold exceeds a maximum observed fluorescence

\# value. Therefore, we used try() to allow an error-recovery.

th. cycle <- try(th.cyc(qPCR $[, 1]$, res, $r=10$, linear $=$ FALSE) $[1]$, silent $=$ TRUE)

\# In addition we used logical statements, which define if a

cq <- ifelse(is(th.cycle, "try-error"), as.numeric(th.cycle), NA)

if (th. cycle $>35$ ) cq $<-$ NA 

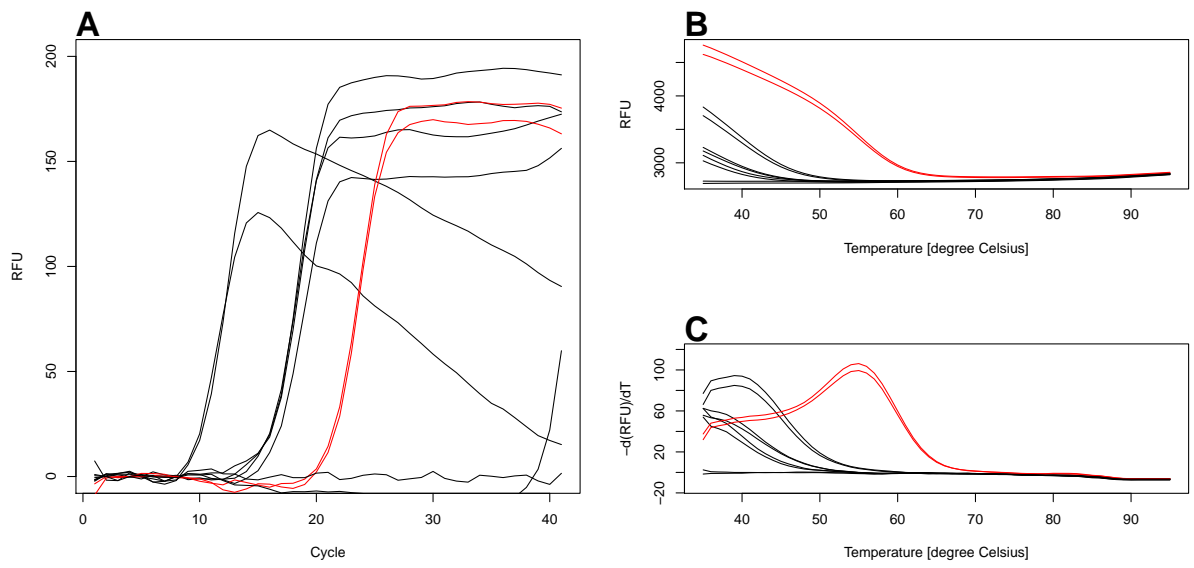

Figure 6: Amplification curve plots and melting curve plots. (A) The raw amplification curve data were pre-processed with the CPP function prior to visualization. To calculate the $T_{M}$ values from the raw melting curve data (B) we used the diffQ function from the MBmca package. (C) We adjusted our algorithm to plot the true positive melting peaks in red, while negative melting peaks were labelled in black. The inspection of the plot and the output of results.tab showed that only D07_katG 315_unkn_EvaGreen (-) and D08_katG 315_unkn_EvaGreen (-) are true positive. RFU, relative fluorescence units; $-\mathrm{d}(\mathrm{RFU}) / \mathrm{dT}$, negative first derivative of the melting-curve.

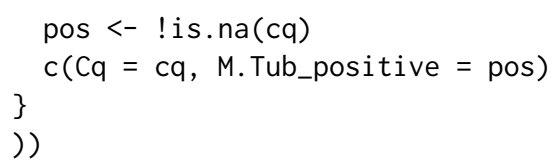

So far we were able to calculate the Cq values for all samples. Next, we performed a melting curve analysis to characterize our samples. Therefore, we fetched the melting curve data from the BioRad object. We focused on the central steps of the melting curve analysis. A comprehensive overview on such analysis was presented in Rödiger et al. (2013a).

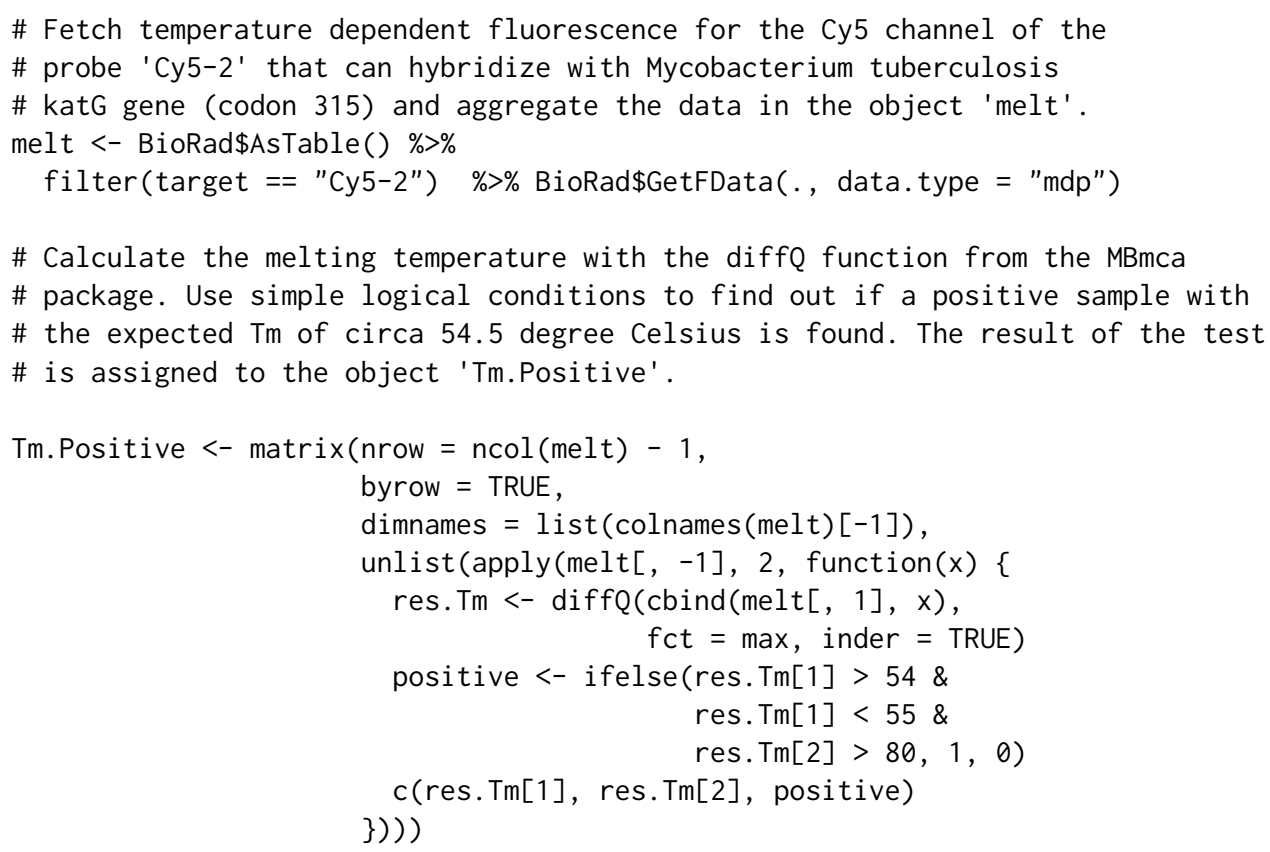

An important part of an automatized report generation is the automatic assignment of decisions (e.g., sample is a wild-type or mutant). Therefore, we used again a simple logic.

\# Present the results in a tabular output as data.frame 'results.tab'.

\# Result of analysis logic is:

\# Cq.Positive \&\& Tm.Positive = Wild-type

\# Cq.Positive \&\& !Tm.Positive = Mutant

\# !Cq.Positive \&\& !Tm.Positive $=$ NTC 


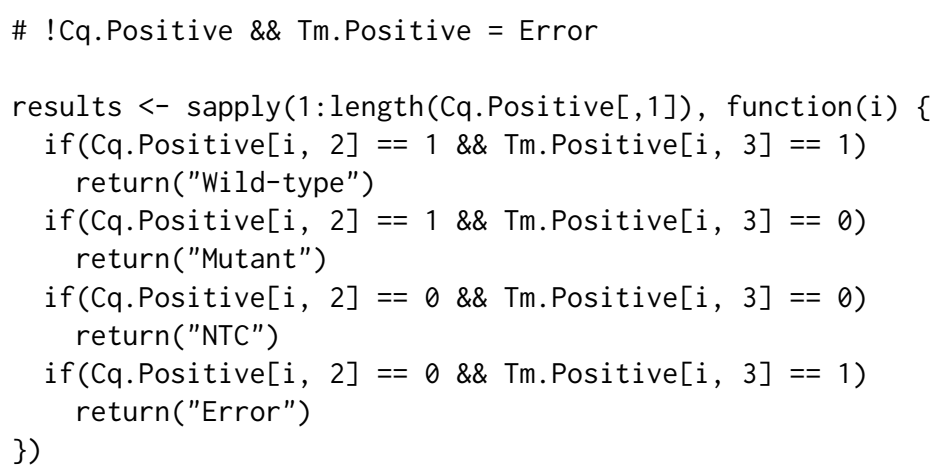

We applied names of variables and factors to all the objects (e.g, Tm. Positive, Cq.Positive) and aggregated them in the object results. tab. The aim of doing this is to present the result in an easy readable form.

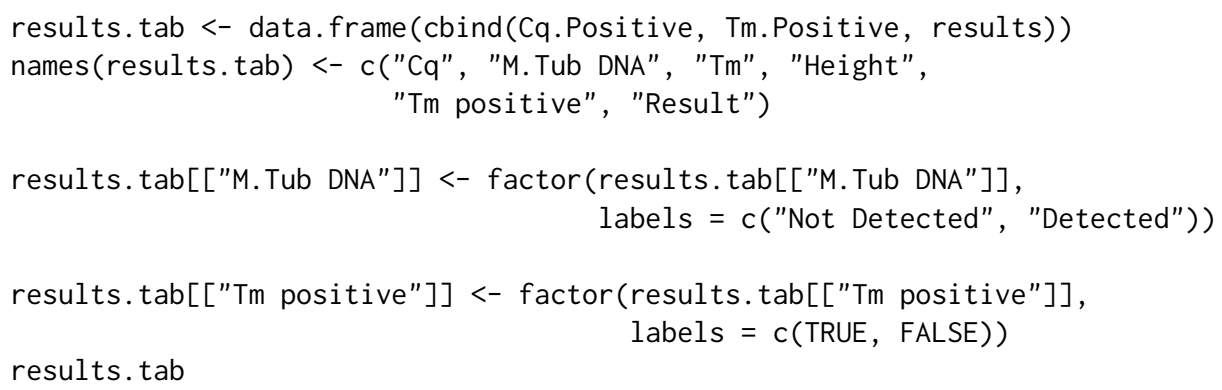

The results of the analysis can be invoked by the statement results. tab (not shown). Finally, we plotted and printed the output of our melting curve (Figure 6B) and melting peak (Figure 6C) analysis.

\# Convert the decision from the 'results' object in a colour code:

\# Negative, black; Positive, red.

color $<-c($ Tm.Positive $[, 3]+1)$

\# Arrange the results of the calculations in a plot.

layout (matrix $(c(1,2,1,3), 2,2$, byrow = TRUE $))$

\# Use the CPP function to preporcess the amplification curve data.

$\operatorname{plot}(N A, N A, x \lim =c(1,41), y \lim =c(0,200), x l a b=$ "Cycle", ylab = "RFU")

mtext ("A", cex $=2$, side $=3$, adj $=0$, font $=2$ )

lapply (2L:ncol(qPCR), function(i)

lines (qPCR $[, 1]$,

CPP(qPCR $[, 1]$, gPCR $[$, i], trans = TRUE,

bg. range $=c(1,9))[[" y \cdot$ norm" $]]$,

col $=\operatorname{color}[i-1]))$

$\operatorname{matplot}(\operatorname{melt}[, 1], \operatorname{melt}[,-1]$, type $=" 1 "$, col $=$ color,

1 ty $=1, x l a b=$ "Temperature [degree Celsius]", ylab = "RFU")

$\operatorname{mtext}(" B ", \operatorname{cex}=2$, side $=3, \operatorname{adj}=0$, font $=2$ )

$\operatorname{plot}(N A, N A, x \lim =c(35,95), y \lim =c(-15,120)$,

$\mathrm{xlab}=$ "Temperature [degree Celsius]",

$\mathrm{ylab}="-\mathrm{d}(\mathrm{RFU}) / \mathrm{dT} ")$

$\operatorname{mtext}\left(" C^{\prime}\right.$, cex $=2$, side $=3$, adj $=0$, font $=2$ )

invisible(

lapply(2L:ncol(melt), function(i)

lines(diffQ(cbind(melt[, 1], melt $[, i])$, verbose $=$ TRUE, $f c t=\max$, inder $=\operatorname{TRUE})[[" x y "]], \operatorname{col}=\operatorname{color}[i-1]))$

)

According to the analysis by MCA the samples 'D07_katG315...' and 'D08_katG315...' were the only positive samples. The remaining samples appeared to be positive in the amplification plot in Figure 5 due to primer-dimer formation or sample contamination. 


\section{Case study three - Isothermal amplification}

Isothermal amplification is an alternative nucleic acid amplification method, which uses a constant temperature rather than cycling through denaturation, annealing and extension steps (Rödiger et al., 2014). The signal is monitored continuously on a time-wise basis. In qIA the abscissa values are often not uniformly spaced. Our CPP function gives a warning in such a case. In qIA the $C q$ values are dependent on the time instead of cycles. In this study we used the th.cyc function from the chipPCR package to determine the time $\left(C q_{t}\right)$ required to reach a defined threshold signal level.

We performed a quantitative isothermal amplification (qIA) with the plasmid $p$ CNG1 by using a Helicase dependent amplification (HDA). Our previously reported VideoScan platform (Rödiger et al., 2013b) was used to control the temperature and to monitor the amplification reaction. The VideoScan technology is based on a highly versatile fluorescence microscope imaging platform, which can be operated with a heating/cooling unit (HCU) for qPCR and MCA applications (Rödiger et al., 2013a,b; Spiess et al., 2015). Since the enzyme DNA Helicase unwinds DNA, no thermal denaturation is needed. HDA conditions were taken from the 'IsoAmp III Universal tHDA Kit', Biohelix Corp, as described by the vendor. In detail, the reaction was composed of 'mix A' $10 \mu \mathrm{L}$ A. bidest, $1.25 \mu \mathrm{L} 10 \mathrm{X}$ buffer, $0.75 \mu \mathrm{L}$ primer ( $150 \mathrm{nM}$ final), $0.5 \mu \mathrm{L}$ template plasmid. Preincubation: The mixture was incubated for $2 \mathrm{~min}$ at $95^{\circ} \mathrm{C}$ and immediately placed on ice. Reaction 'mix B' contained $5 \mu \mathrm{L}$ A. bidest., $1.25 \mu \mathrm{L} \mathrm{10X} \mathrm{buffer,}$ $2 \mu \mathrm{L} \mathrm{NaCl}, 1.25 \mu \mathrm{L} \mathrm{MgSO}_{4}, 1.75 \mu \mathrm{L}$ dNTPs, $0.25 \mu \mathrm{L}$ EvaGreen (Biotium), $1 \mu \mathrm{L}$ enzyme mix. The mix was covered with $50 \mu \mathrm{L}$ mineral oil (Roth). The fluorescence measurement in VideoScan HCU started directly after adding 'mix $\mathrm{B}^{\prime}$ at $65^{\circ} \mathrm{C}$. A $1 x$ (D1) and a $1: 10$ dilution (D2) were tested. The resulting dataset $\mathrm{C} 81$ is part of the chipPCR package. Two concentrations (stock and 1:10 diluted stock) of input DNA were used in the HDA. First, we had a look at the C81 dataset with the str function.

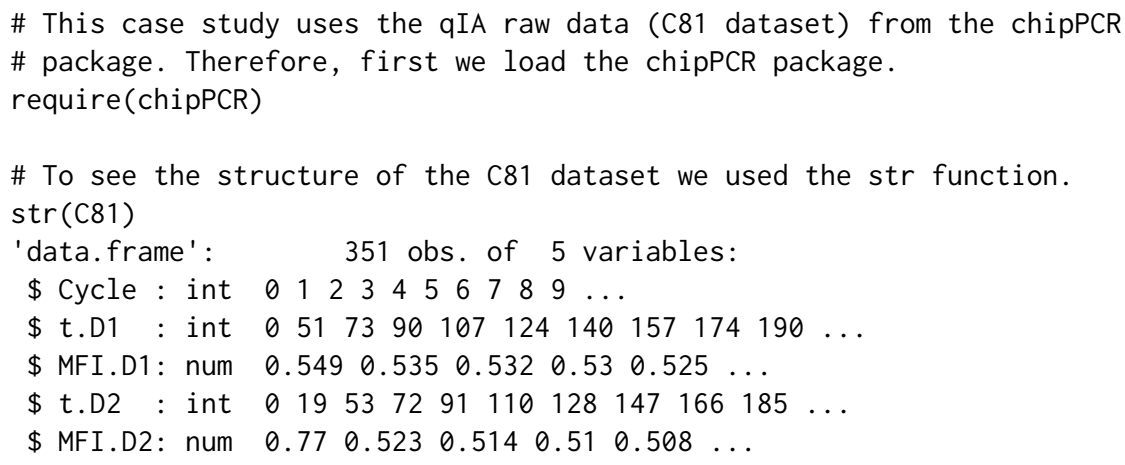

C81 is a data frame. The first column contains the measuring points ( $\mathrm{Cycle}$ ) and the consecutive columns contain the time stamps (e.g., t.D1, in seconds according to the chipPCR manual) and the signal hight (MFI.D1, as mean fluorescence intensity; MFI). A brief look at the time stamps showed that the data are not uniformly spaced. Warning messages by the CPP functions are just a reminder for the user to take care during the pre-processing. Methods like smoothing might cause artifacts (Spiess et al., 2015). We know that the HDA is a time-dependent reaction. Therefore, we do not have a use for the discrete measuring points. Next, we proceeded with the analysis. Similar to the previous case studies, we prepared the plot of the raw data. Since the raw data showed a slight negative trend and an off-set of circa $0.45 \mathrm{MFI}$ (mean fluorescence intensity) it was necessary to pre-process the raw data (Figure 7A).

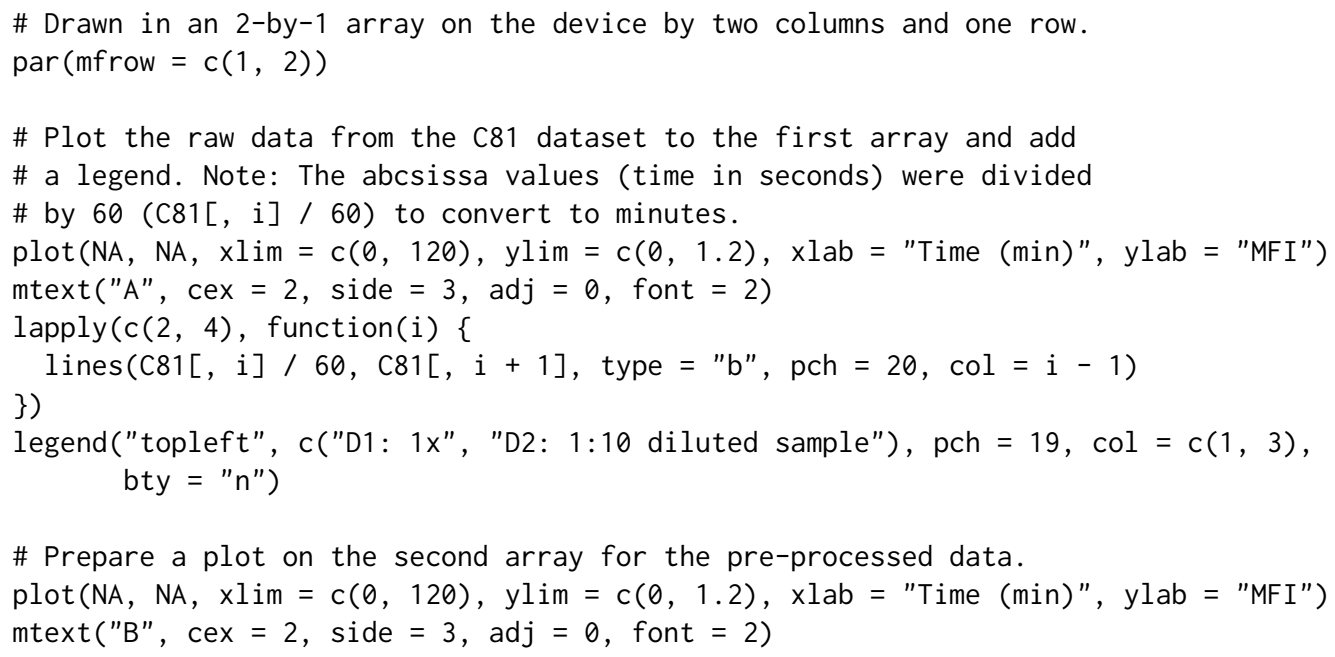



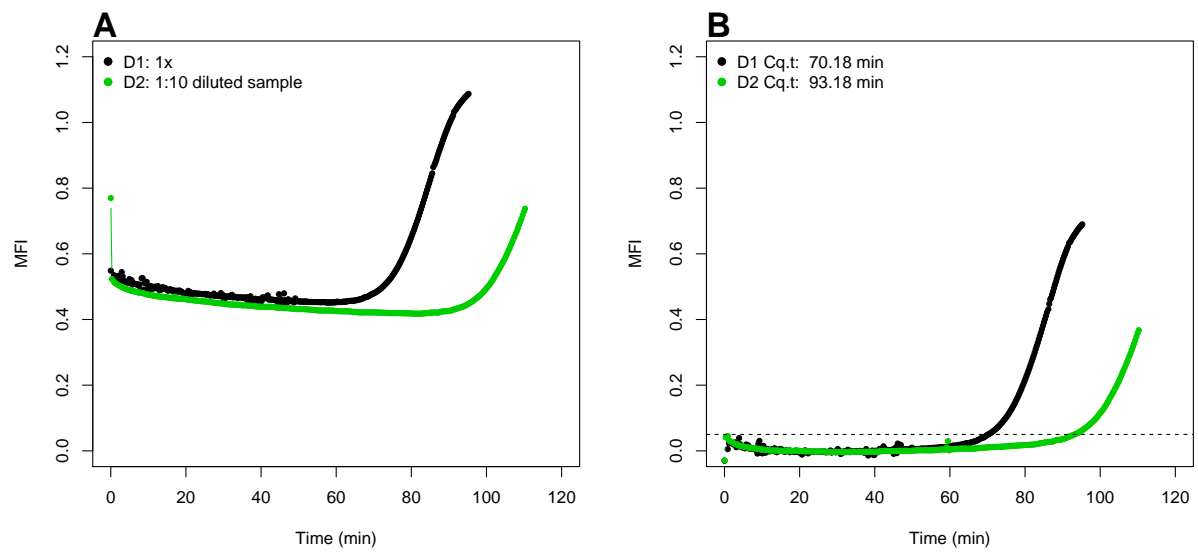

Figure 7: Quantitative isothermal amplification by Helicase dependent amplification (HDA). (A) The raw data of the HDA (D1, undiluted; D2, $1: 10$ diluted) exhibit some outliers (detector artifacts), an off-set of circa $0.5 \mathrm{MFI}$ and a slight negative trend in the baseline region (0-52 minutes). (B) We used the CPP function to smooth the data with a spline function. Baselining was done with a linear regression model (robust MM-estimator). Finally, we used the th. cyc function (chipPCR) to calculate the cycle threshold time for samples D1 and D2. The threshold value was set to $r=0.05$ (- - threshold line). $C q_{t}$, required time to reach a defined threshold signal level. MFI, mean fluorescence intensity.

First, we used the CPP function to pre-process the raw data. Similar to the other case studies we baselined and smoothed the amplification curve data prior to the analysis of the of the $C q_{t}$ value. However, instead of the Savitzky-Golay smoother we used a cubic spline (method = "spline") in the CPP function. In addition, outliers were automatically removed in the baseline region (Figure 7A and B). The background range was defined by bare eye to be between the $1^{\text {st }}$ and $190^{\text {th }}$ data point (corresponding to a baseline region between 0 and 52 minutes). The CPP uses a linear least squares or robust fit methods (e.g., Rfit by Kloke and McKean 2012) to estimate the slope of the background (Rödiger et al., in press).

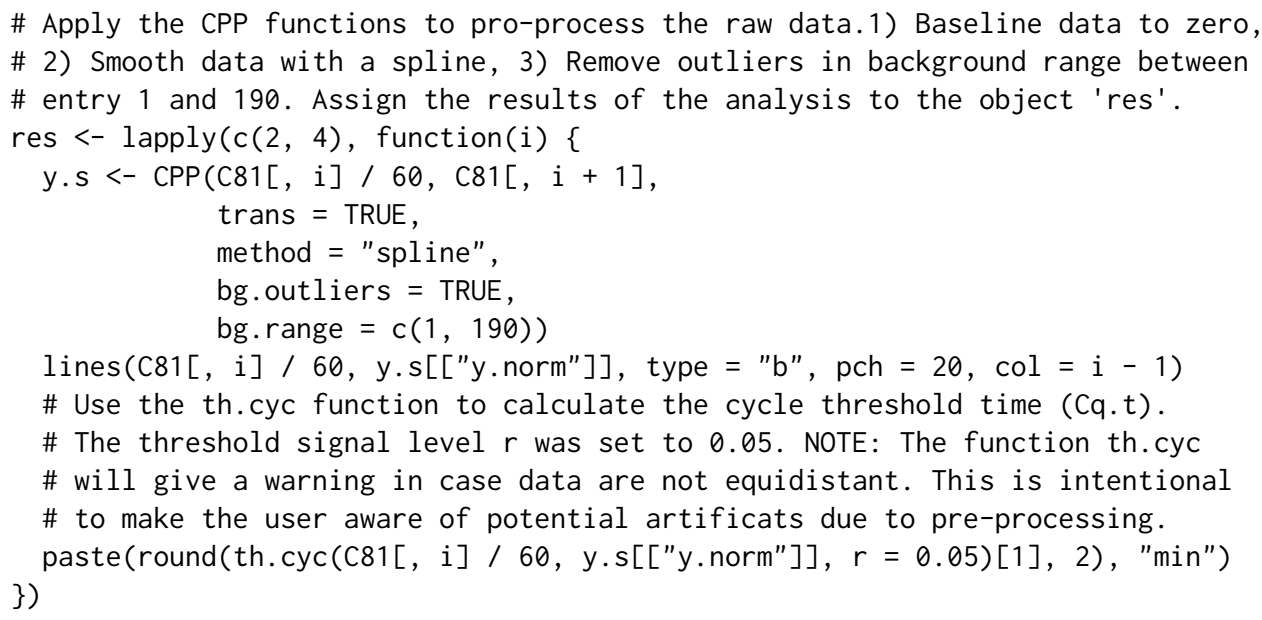

\# Add the cycle threshold time from the object 'res' to the plot.

abline $(\mathrm{h}=0.05$, lty $=2)$

legend("topleft", paste(c("D1 Cq.t: ", "D2 Cq.t: "), res), pch = 19, col $=c(1,3)$, bty $=" n ")$

The pre-processed data were subjected to the analysis of the $C q_{t}$ values. It is important to note that the trend correction and proper baseline was a requirement for a sound calculation. We calculated $C q_{t}$ values of 70.18 minutes and 93.18 minutes for the stock (D1) and 1:10 (D2) diluted samples, receptively.

\section{Case study four - Digital PCR}

We have developed the dpcR package for analysis and presentation of digital PCR experiments. This package can be used to build custom-made analysis pipelines and provides structures to be 
openly extended by the scientific community. Simulations and predictions of binomial and Poisson distributions, commonly used theoretical models of dPCR, statistical data analysis methods, plotting facilities and report generation tools are part of the package (Pabinger et al., 2014). Here, we show a case study for the $\mathbf{d p c R}$ package. Simulations are part in many educational curricula and greatly support teaching. In this case study, we mimicked an in silico experiment for a droplet digital PCR experiment similar to Figure 1. The aim was to assess the concentration of the template sample. In the following we will use the expression partition as synonym for droplet. The number of positive partitions $(k)$, total number of partitions $(n)$ and the size of the partition are the only data required for the analysis. The estimate of the mean number of template molecules per partition $(\hat{\lambda})$ was calculated using the following equation (Huggett et al., 2013):

$$
\hat{\lambda}=-\ln \left(1-\frac{k}{n}\right)
$$

The average partition volume in our experiment was assumed to be $5 \mathrm{~nL}$. We counted $n=16800$ partitions in total from which $k=4601$ partitions were positive. The binomial distribution of positive and negative partitions was used to determine $\hat{\lambda}$ (Figure 8). Our package allows both easy estimation of a density of the parameter and calculation of confidence intervals (CI) using Wilson's method (Brown et al., 2001) at a confidence interval level of 0.95. The true number of template molecules per partition $(\hat{\lambda})$ is likely to be included in the range of the CI (Milbury et al., 2014). The obtained mean number of template molecules per partition multiplied by the volume of the partitions $(16800 \cdot 5 \mathrm{~nL})$ constitutes the sample concentration.

\# Load the dpcR package for the analysis of the digital PCR experiment. require $(\mathrm{dpcR})$

\# Analysis of a digital PCR experiment. The density estimation.

\# In our in-silico experiment we counted in total 16800 partitions (n).

\# Thereof, 4601 were positive (k).

$\mathrm{k}<-4601$

$\mathrm{n}<-16800$

(dens <- dpcr_density $(k=k, n=n$, average = TRUE, methods = "wilson", conf.level $=0.95)$ )

legend("topleft", paste("k:", k,"\nn:", n))

\#dev. off ()

\# Let us assume, that every partition has roughly a volume of $5 \mathrm{~nL}$.

\# The total concentration (and its confidence interval) in molecules/ml is

\# (the factor $1 \mathrm{e}-6$ is used for the conversion from $\mathrm{nL}$ to $\mathrm{mL}$ ):

dens[4:6] / $5 * 1 \mathrm{e}-6$

Since we assumed a partition volume of $5 \mathrm{~nL}$ we have a total volume of $0.084 \mathrm{~mL}$ and $6.40 \times 10^{-8}$ (95\% CI: $\left.6.2 \times 10^{-8}-6.6 \times 10^{-8}\right)$ molecules $/ \mathrm{mL}$ in the sample.

Selected functionality was implemented as an interactive shiny (Chang et al., 2015) GUI application to make the software accessible for users who are not fluent in $\mathrm{R}$ and for experts who wish to automatize routine tasks. Details and examples of the shiny web application framework for $\mathrm{R}$ can be found at $\mathrm{http}$ : //shiny . rstudio.com/. We implemented flexible user interfaces, which run the analyses and graphical representation into interactive web applications either as service on a web server or on a local machine without knowledge of HTML or ECMAScript (see the dpcR manual). The interface is designed in a cascade workflow approach (Data import $\rightarrow$ Analysis $\rightarrow$ Output $\rightarrow$ Export) with interactive user choices on input data, methods and parameters using typical GUI elements such as sliders, dropdowns and text fields. An example can be found at https://michbur. shinyapps.io/dpcr_density/. This approach enables the automatized output of $\mathrm{R}$ objects in combined plots, tables and summaries.

\section{Case study five - Digital PCR partition volume correction}

There is an ongoing debate in the scientific community about the effect of the partition volume on the estimated copy numbers size (Huggett et al., 2015a; Corbisier et al., 2015; Majumdar et al., 2015). Corbisier et al. (2015) showed that the partition volume is a critical parameter for the measurement of copy number concentrations. Their study revealed that the average droplet volume defined in the QuantaSoft software (v. 1.3.2.0, BioRad QX100 Droplet Digital PCR System) is $8 \%$ lower than the real volume. In consequence, results of quantifications were systematically biased between different dPCR platforms. Case study four served as an introduction into the analysis of simulated dPCR experiments with the dpcR package. In the next case study, number five, we used the pds_raw dataset, which was generated by a BioRad QX100 Droplet Digital PCR System experiment. We re-analysed the data with 


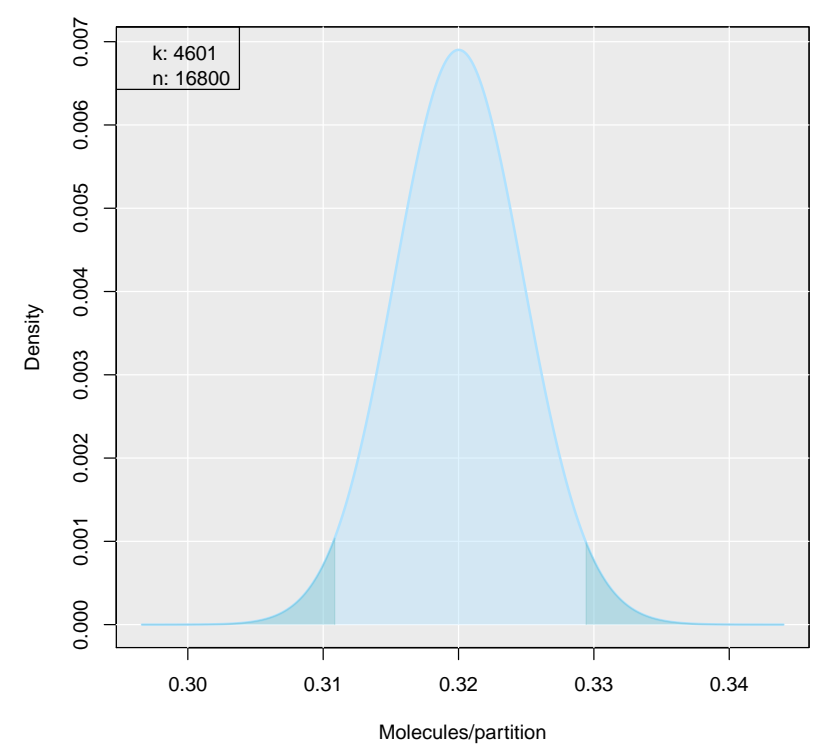

Figure 8: dpcr_density function from the dpcR package used for analysis of a droplet digital PCR experiment. From 16800 counted partitions $(n) 4601$ were positive $(k)$. The chart presents the distribution of mean number of template molecules per partition $(\hat{\lambda}) . n$, number of total partitions; $k$, number of positive partitions.

the partition volume of $0.834 \mathrm{~nL}$ as proposed by Corbisier et al. (2015) and a volume of $0.90072 \mathrm{~nL}$ as used in the BioRad QX100 Droplet Digital PCR System.

Our experimental setup was as follows. A duplex assay was used to simultaneously detect a constant amount of genomic DNA (theoretically $10^{2}$ copies $/ \mu \mathrm{L}$ ) and a variable amount of plasmid DNA (10-fold serial dilution, not shown). The genomic DNA was isolated from Pseudomonas putida KT2440 and the plasmid was $p$ COM10-StyA::EGFP StyB. Template DNA was heat treated at $95^{\circ} \mathrm{C}$ for 5 min prior to PCR. We detected in Channel 1 the genomic DNA marker ileS with FAM labelled Taqman probes and in Channel 2 the plasmid DNA marker styA with HEX labelled Taqman probes. All primer/probe sequences and experimental condiditions are described in Jahn et al. 2013, 2014 and the manual of the $\mathbf{d p c R}$ package. Gating and partition clustering was taken without any modification from the data output of the BioRad QX100 Droplet Digital PCR System. Each partition is represented by a dot in Figure 9. First, we had a look at the data structure of the pds_raw dataset.

\# Load the dpcR package and use the pds_raw dataset for the analysis of the

\# digital PCR experiment.

\# To get an overview of the data set we used the head and summary $R$ functions

\# in a chain. The output shows that the dataset contains lists of different

\# samples $(A 01 \ldots)$

require $(\mathrm{dpcR})$

$\operatorname{head}($ summary (pds_raw))

$\begin{array}{ll}\text { Length Class } & \text { Mode } \\ \text { A01 "3" } & \text { "data.frame" "list" } \\ \text { A02 "3" } & \text { "data.frame" "list" } \\ \text { A03 "3" } & \text { "data.frame" "list" } \\ \text { A04 "3" } & \text { "data.frame" "list" } \\ \text { B01 "3" } & \text { "data.frame" "list" } \\ \text { B02 "3" } & \text { "data.frame" "list" }\end{array}$

\# Next, we used str for the element A01. The element of the list contains a data frame \# with three columns. Two contain amplitude values (fluorescence intensity) and one \# contains cluster results (integer values of $1-4$ ).

$\operatorname{str}($ pds_raw[["A01"]])

'data.frame': 11964 obs. of 3 variables:

\$ Assay1.Amplitude: num $397399402416417 \ldots$ 
\$ Assay2. Amplitude: num $37323808400737783685 \ldots$

\$Cluster : int $\begin{array}{llllllllllll}4 & 4 & 4 & 4 & 4 & 4 & 4 & 4 & 4 & 4 & \ldots\end{array}$

Since the structure of the dataset was known now we selected samples for the analysis. According to the dpcR manual, the samples A02, B02, C02 and D02 contained the values for the replicates at a 1: 100 dilution and G04 contained the values for the non-template control.

\# Select the wells for the analysis. A02 to D02 are four replicate dPCR reactions \# and G04 is the no template control (NTC) (see dpcR manual for details).

wells <- c("A02", "B02", "CO2", "D02", "G04")

\# Set the arrangement for the plots. The first column contains the amplitude \# plots, column two the density functions and column three the concentration \# calculated according to the droplet volume as defined in the QX100 system, \# or the method proposed by Corbisier et al. (2015).

$\operatorname{par}($ mfrow $=c(5,3))$

\# The function bioamp was used in a loop to extract the number of positive and \# negative partitions from the sample files. The results were assigned to the \# object 'res' and plotted.

for (i in $1 \mathrm{~L}:$ length(wells)) \{

cluster.info <- unique(pds_raw[wells[i]][[1]]["Cluster"])

res $<-\operatorname{bioamp}\left(d a t a=p d s \_r a w[w e l l s[i]][[1]]\right.$, amp_x $=2$, amp_y $=1$, main = paste $(" W e l l "$, wells $[i]), x l a b=$ "Amplitude of ileS (FAM)", $\mathrm{ylab}=$ "Amplitude of styA (HEX)", $\mathrm{xlim}=\mathrm{c}(500,4700)$, ylim $=c(0,3300)$, pch $=19)$

legend("topright", as.character(cluster.info[, 1]), col = cluster.info[, 1], pch $=19)$

Next, we used the results from the object res to get the information about the number of positive partitions for the plasmid DNA marker styA. This is to be found in the clusters 2 and 3.

\# Counts for the positive clusters 2 and 3 were assigned to new objects $\#$ and further used by the function dpcr_density to calculate the number \# of molecules per partition and the confidence intervals. The results \# were plotted as density plot.

k. tmp <- $\operatorname{sum}($ res[1, "Cluster.2"], res[1, "Cluster.3"])

\# Counts for all clusters

n. $\operatorname{tmp}<-\operatorname{sum}(\operatorname{res}[1]$,

\# Our next line is used to limit the $x$-axis of the plot to a meaningful range. if $(i<5) x . \lim <-c(0.065,0.115)$ else $x . \lim <-c(0,0.115)$

\# The next step is the calculation of the dPCR statistics.

dens <- dpcr_density $(k=k . t m p, n=n . t m p$, average = TRUE, methods = "wilson", conf.level $=0.95, x \lim =x . \lim$, bars $=$ FALSE)

legend("topright", paste("k:", k.tmp,"\nn:", n.tmp), bty = "n")

\# Finally, the concentration of the molecules was calculated with the volume \# used in the QX100 system and as proposed by Corbisier et al. (2015). The

\# results were added as barplot with the confidence intervals.

res.conc $<-$ rbind(original $=$ dens $[4: 6] / 0.90072$,

corrected $=$ dens $[4: 6] / 0.834)$

barplot(res.conc [, 1], col = c("white", "grey"),

names = c("Bio-Rad", "Corbisier"),

main = "Influence of $\backslash$ DDroplet size", ylab = "molecules $/ \mathrm{nL}$ ", $\mathrm{ylim}=\mathrm{c}(0,1.5 * 10 \mathrm{E}-2))$

$\operatorname{arrows}(c(0.7,1.9)$, res.conc $[, 2], c(0.7,1.9)$, res.conc $[, 3]$, angle $=90$, code $=3$, lwd $=2$ ) 

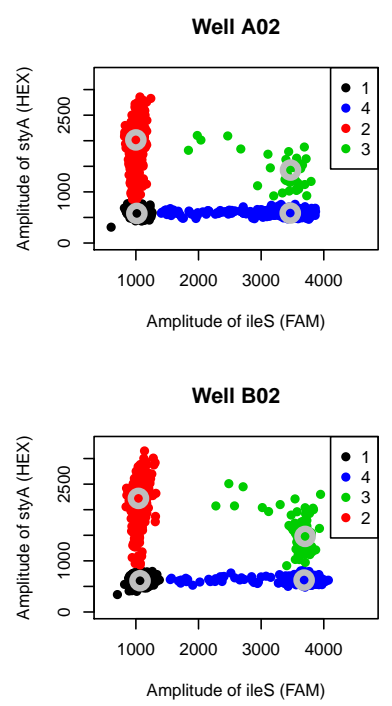

Well C02

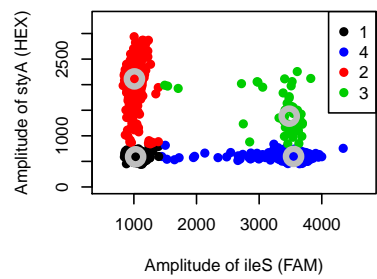

Well D02

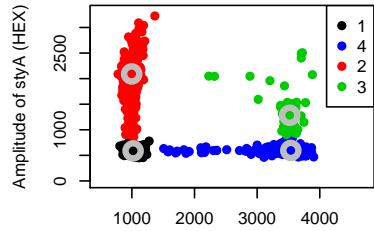

Amplitude of ileS (FAM)

Well G04

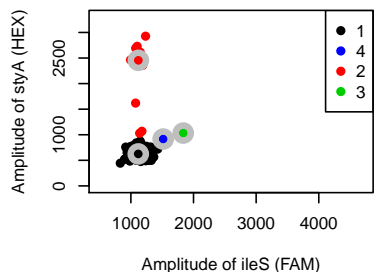

Number of molecules per partition

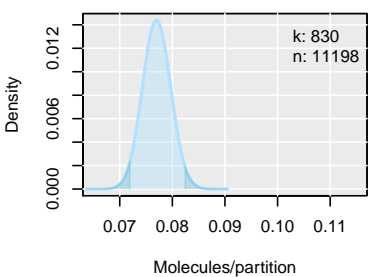

Number of molecules per partition

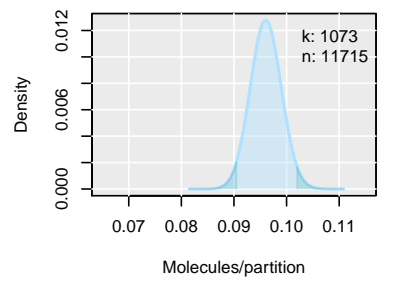

Number of molecules per partition

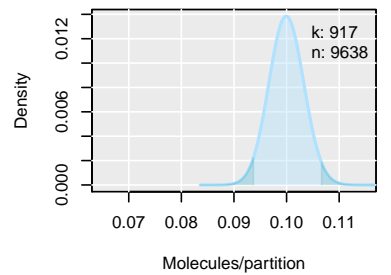

Number of molecules per partition

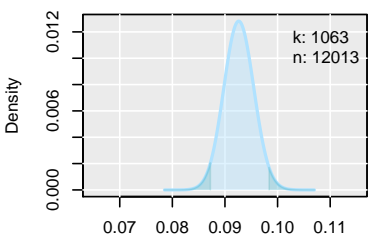

Molecules/partition

Number of molecules per partition

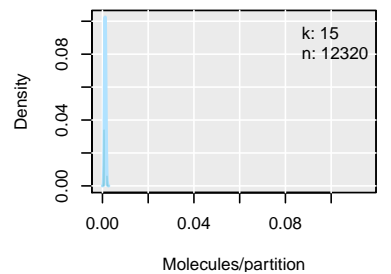

Influence of

Droplet size

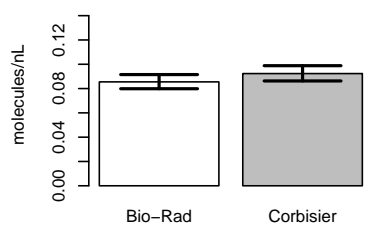

Influence of

Droplet size

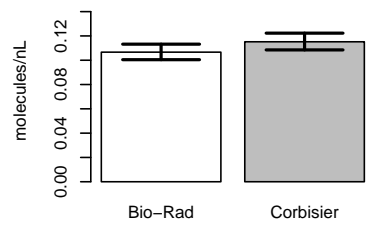

Influence of Droplet size

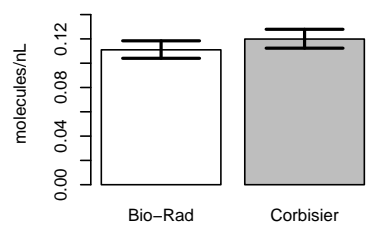

Influence of Droplet size

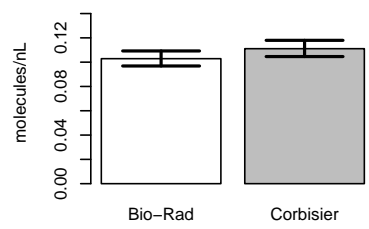

Influence of Droplet size

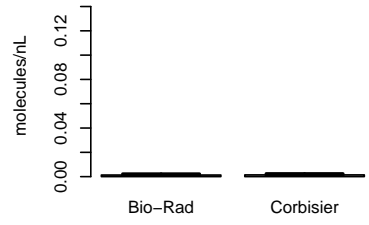

Figure 9: Analysis of a droplet digital PCR experiment. We used the pds_raw dataset from the dpcR package. All raw data were generated with a BioRad QX100 Droplet Digital PCR System. Column one: Amplitude plot of raw data shown by the bioamp function. Each dot represents a partition in a cluster. Cluster $1 \bullet$, Cluster $2 \bullet=$ Target, Cluster $3 \bullet=$ Target, Cluster $4 \bullet$. A02-D04 are replicate measurements and well G04 is a negative control; Column two: Density function with showing the number of molecules per partition. All replicates had similar numbers of counted positive partitions $(k)$ and total number of partitions (n); Column three: Concentration of DNA molecules based on the volume used by the BioRad QX100 Droplet Digital PCR System and the volume proposed by Corbisier et al. (2015). $n$, number of total partitions; $k$, number of positive partitions; sty $A$, styrene monooxygenase; ileS, isoleucyl-tRNA synthetase; FAM, Fluorescein channel; HEX, hexachlorofluorescein.

As proposed by Corbisier et al. (2015) the analysis with the non-corrected droplet volume results in an underestimation of sample concentrations (Figure 9 column 3). Our results show, that the replicates in Figure 9 A02 - D02 vary. However, the variation appeared to be within a similar range (Figure 9 column 2). The positive samples (Figure 9 A02-D02) clearly differ from the negative control 
(Figure 9 G04). To compare this we performed a statistical test. This is basically a comparison of proportions as described elsewhere (Wang and Shan, 2013). Statistical capabilities of R simplify the next steps of the analysis of digital PCR experiments. The dpcR package offers a flexible wrapper test_counts around several methods of comparing results of several reactions. In the following, we used a test implemented in the rateratio.test package by Fay (2010).

\# The first step is as usual extracting data and shaping it into an object of

\# appropriate class, in this case 'ddpcr' (droplet digital PCR)

cluster_data <- do.call(bind_dpcr, lapply(1L:length(wells), function(i) \{

cluster.info <- unique(pds_raw[wells[i]][[1]]["Cluster"])

res $<-$ bioamp $($ data $=$ pds_raw[wells[i]][[1]], amp_x $=2$, amp_y $=1$, plot $=$ FALSE)

\# create ddpcr object for each experiment

create_dpcr $($ data $=c(\operatorname{rep}(1, \operatorname{res}[1, " C l u s t e r .2 "]), \operatorname{rep}(1, \operatorname{res}[1, " C l u s t e r .3 "]))$, $\mathrm{n}=$ as.integer $(\operatorname{sum}(\operatorname{res}[1])$,$) , threshold =1$,

\})) type $=$ "np", adpcr $=$ FALSE)

\# Message: 'Different number of partitions.' is expected while joining objects

\# with uneven length as droplet-based experiments. The message is specifically

\# verbose to also deliver that the bind_dpcr function does not

\# recycle shorter vectors to prevent the addition of non-existent data points.

\# Give experiments proper names.

colnames(cluster_data) <- wells

\# We choose the ratio model, which uses multiple ratio tests from the 'rateratio.test'

\# package.

comp <- test_counts(cluster_data, model = "ratio")

Output format of test_counts is familiar to all $\mathrm{R}$ users:

$>$ comp

Groups:

group lambda lambda.low lambda.up

$\begin{array}{llllll}\text { A02 } & \text { b } 0.077011051 & 0.0704320674 & 0.084179123\end{array}$

B02 c c $0.096061636 \quad 0.0888030003 \quad 0.103883369$

$\begin{array}{llllll}C 02 & c & 0.099979708 & 0.0918318731 & 0.108811947\end{array}$

$\begin{array}{llllll}\text { D02 } & \text { c } & 0.092649940 & 0.0856180946 & 0.100230919\end{array}$

$\begin{array}{lllllll}\text { G04 a } & 0.001218274 & 0.0006348818 & 0.002337119\end{array}$

Results of multiple comparison:

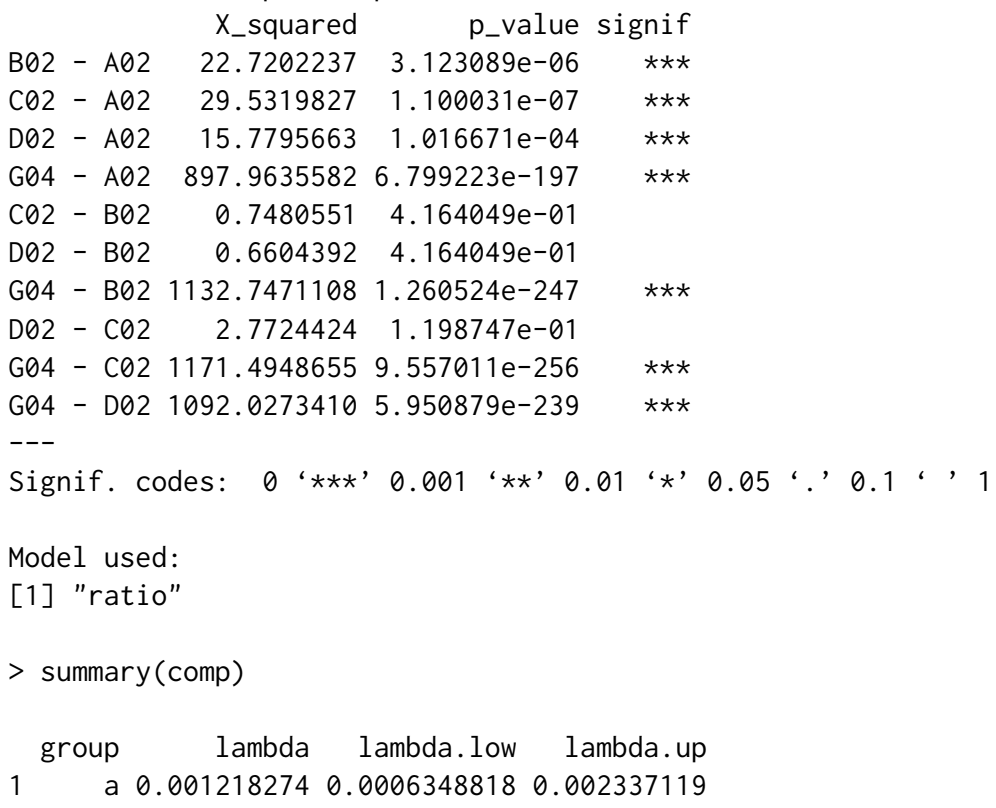




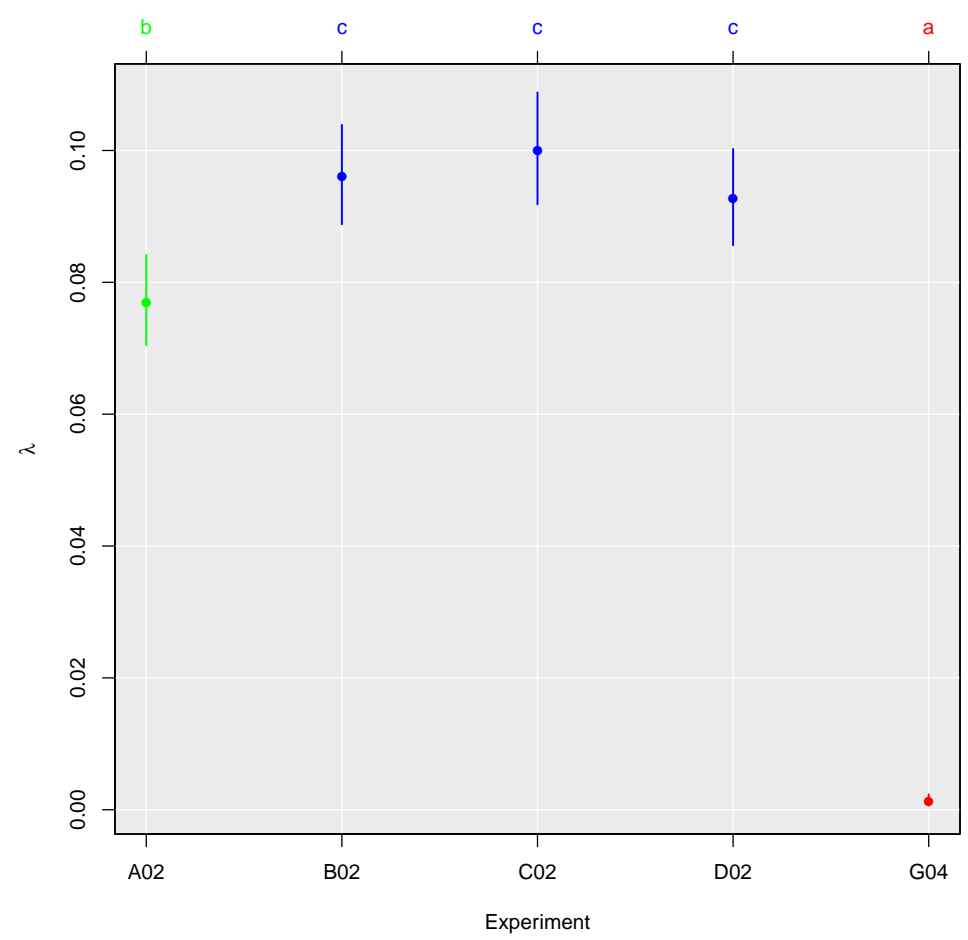

Figure 10: Comparison of droplet digital PCR runs. The plot method of the test_counts function from the $\mathbf{d p c R}$ package was used. The experiments were aggregated into colour coded groups based on their estimated mean number of copies per partition $(\lambda)$. A02 remained individual b, B02, C02 and D02 formed a group $\mathrm{c}$ and the negative control also remained individual a.

The result of the analysis suggests that replicate samples B02, C02 and D02, with exception of A02, do not differ significantly. The negative control G04 was totally different from the other samples (Figure 10). Our dpcR package can be used to analyse data beyond a level as provided by commercial software. Access to the whole R environment allows performing more elaborated research in a highly customized workflow. In conclusion, our case study shows, that the R environment can be used to circumvent problems of locked-in systems.

\section{Discussion and conclusion}

This study gave a brief introduction to the analysis of qPCR, qIA, MCA and dPCR experiments with $R$. In addition to this, we briefly referenced to a vast collection of additional packages available from CRAN and Bioconductor. The packages may be considered as the building blocks (libraries) to create what users want and need. We showed that automatized research with $\mathrm{R}$ offers powerful means for statistical analysis and visualization. This software is not tied to a vendor or application (e.g., chamber or droplet based digital PCR, capillary or plate qPCR). It should be quite easy even for an inexperienced user to define a workflow and to set up a cross-platform environment for specific needs in a broad range of technical settings (Figure 11). This environment enforces no monolithic integration. We claim that the modular structure allows the user to perform flexible data analysis, adjusted to their needs and to design frameworks for high-throughput analysis. Furthermore, R enables the users to access and reuse code for the creation of reports in various formats (e.g., HTML, PDF).

Despite the fact that $\mathrm{R}$ is free of charge, it is quite possible to build commercial applications. The packages cover implementation of novel approaches and peer-reviewed analysis methods. $R$ packages are an open environment to adopt to the growing knowledge in life sciences and medical sciences. Therefore, we argue that this environment may provide a structure for standardized nomenclature and serve as reference in qPCR and dPCR analysis. Speaking about openness, it needs to be emphasized that the main advantage of this software is its transparency at any time for anybody. Thus, it is possible to track numerical errors. A disadvantage of $\mathrm{R}$ is the lack of comprehensive GUIs for qPCR analysis. GUIs are key technologies to spread the use of $\mathrm{R}$ in bioanalytical sciences. Currently, we are establishing the 'pcRuniveRsum' (http://michbur.github.io/pcRuniveRsum/) as an on-line resource for the interested users. The command-line structure makes $R$ 'inaccessible' for many novices. We try 

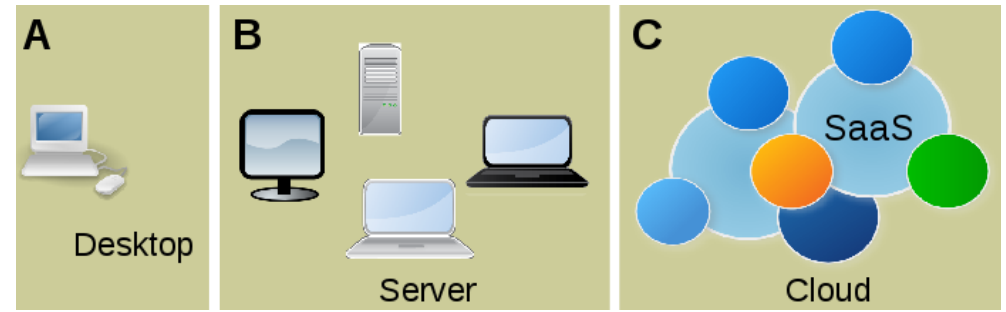

Figure 11: Deployment of $R$ applications for the $\mathrm{GPCR}$ and $\mathrm{dPCR}$ experiments. (A) R is typically run from a desktop computer and operated by a GUI/IDE application such as RStudio or RKWard. This approach provides a flexible workflow for individuals. (B) Another approach is to run $\mathrm{R}$ with specific applications on a local server. Such scenarios are useful for the deployment within research departments or cooperate units (Nolan and Temple, 2014). (C) Cloud computing (CC) provides shared and scalable computing capacity (e.g., computing capacity, application software) and storage capacity (e.g., databases) as a service to an individual user or a community. Service categories include: Infrastructure-as-a-Service (IaaS), Platform-as-a-Service (PaaS) and Software-as-a-Service (SaaS) over a network. Providers of CC manage the infrastructure and resources to achieve coherence and economies of scale similar to a utility over a network (i.p., Internet) (Ohri, 2014).

to solve this problem with easily accessible GUIs (Rödiger et al., 2012). However, the work on this additions has been recently started and is still in progress.

\section{Acknowledgment}

Part of this work was funded by the BMBF InnoProfile-Transfer-Projekt 03 IPT 611X, the European Regional Development Fund (ERDF/EFRE) on behalf of the European Union, the Sächsische Aufbaubank (Free State of Saxony, Germany) and the Russian Ministry of Education and Science (project No. RFMEFI62114X0003), with usage of scientific equipment of Center for collective use 'Biotechnology' at All-Russia Research Institute of Agricultural Biotechnology. We would like to thank the R community. We would like to thank Mario Menschikowski (Technical University Dresden) for the droplet digital PCR samples.

\section{Bibliography}

M. G. Almiron, B. Lopes, A. L. C. Oliveira, A. C. Medeiros, and A. C. Frery. On the numerical accuracy of spreadsheets. Journal of Statistical Software, 34(4):1-29, 4 2010. URL http: //www. jstatsoft. org/ v34/i04. [p128]

R. Bååth. The state of naming conventions in R. The R Journal, 4(2):74-75, Dec. 2012. URL http: //journal.r-project.org/archive/2012-2/RJournal_2012-2_Baaaath.pdf. [p129]

P. Biecek and M. Kosinski. archivist: Tools for Storing, Restoring and Searching for R Objects, 2015. URL http://CRAN.R-project.org/package=archivist. R package version 1.3. [p130]

K. A. Blagodatskikh, S. Roediger, and M. Burdukiewicz. RDML: Importing Real-Time Thermo Cycler (qPCR) Data from RDML Format Files, 2015. URL http://CRAN. R-project.org/package=RDML. R package version 0.8-4. [p128]

L. D. Brown, T. T. Cai, and A. Dasgupta. Interval estimation for a binomial proportion. Statistical Science, 16:101-133, 2001. [p140]

M. Burdukiewicz, S. Roediger, and B. Jacobs. dpcR: Digital PCR Analysis, 2015. URL http://CRAN. Rproject. org/package=dpcR. R package version 0.1.4.0. [p127]

P. Burns. Spreadsheet addiction. online, 2014. URL http://web. archive. org/web/20141009042532/ http://www. burns-stat.com/documents/tutorials/spreadsheet-addiction/. [p128]

S. A. Bustin. The reproducibility of biomedical research: Sleepers awake! Biomolecular Detection and Quantification, 2:35-42, Dec. 2014. [p127]

I. Castro-Conde and J. de Uña-Álvarez. sgof: An R package for multiple testing problems. The $R$ Journal, 6(2):96-113, Dec. 2014. [p127] 
W. Chang, J. Cheng, J. J. Allaire, Y. Xie, and J. McPherson. shiny: Web Application Framework for R, 2015. URL http://CRAN. R-project.org/package=shiny. R package version 0.12.1. [p31, 140]

P. Corbisier, L. Pinheiro, S. Mazoua, A.-M. Kortekaas, P. Y. J. Chung, T. Gerganova, G. Roebben, H. Emons, and K. Emslie. DNA copy number concentration measured by digital and droplet digital quantitative PCR using certified reference materials. Analytical and Bioanalytical Chemistry, 407(7): 1831-1840, 2015. [p140, 141, 143]

A. J. Durán, M. Pérez, and J. L. Varona. The misfortunes of a trio of mathematicians using computer algebra systems. Can we trust in them? Notices of the American Mathematical Society, 2014. [p128]

H. Dvinge and P. Bertone. HTqPCR: High-throughput analysis and visualization of quantitative real-time PCR data in R. Bioinformatics, 25(24):3325-3326, Dec. 2009. [p128]

C. Ekstrom, I. M. Skovgaard, and T. Martinussen. kulife: Datasets and Functions from the (Now NonExisting) Faculty of Life Sciences, University of Copenhagen, 2013. URL http: //CRAN. R-project.org/ package=kulife. R package version 0.1-14. [p128]

M. P. Fay. Two-sided exact tests and matching confidence intervals for discrete data. The R Journal, 2 (1):53-58, June 2010. [p144]

C. Gandrud. Reproducible Research with R and RStudio. Chapman and Hall/CRC, July 2013. [p129]

R. C. Gentleman, V. J. Carey, D. M. Bates, B. Bolstad, M. Dettling, S. Dudoit, B. Ellis, L. Gautier, Y. Ge, J. Gentry, K. Hornik, T. Hothorn, W. Huber, S. Iacus, R. Irizarry, F. Leisch, C. Li, M. Maechler, A. J. Rossini, G. Sawitzki, C. Smith, G. Smyth, L. Tierney, J. Y. H. Yang, and J. Zhang. Bioconductor: Open software development for computational biology and bioinformatics. Genome Biology, 5(10):R80, 2004. [p128, 129]

M. Guescini, D. Sisti, M. B. L. Rocchi, L. Stocchi, and V. Stocchi. A new real-time PCR method to overcome significant quantitative inaccuracy due to slight amplification inhibition. BMC Bioinformatics, 9(326), July 2008. [p130]

H. Hofmann, A. Unwin, and D. Cook. Let graphics tell the story - Datasets in R. The R Journal, 5(1): 117-130, June 2013. [p129]

J. Huggett, J. O'Grady, and S. Bustin. How to make mathematics biology's next and better microscope. Biomolecular Detection and Quantification, 1(1):A1-A3, 2014. [p127]

J. F. Huggett, C. A. Foy, V. Benes, K. Emslie, J. A. Garson, R. Haynes, J. Hellemans, M. Kubista, R. D. Mueller, T. Nolan, M. W. Pfaffl, G. L. Shipley, J. Vandesompele, C. T. Wittwer, and S. A. Bustin. The digital MIQE guidelines: Minimum information for publication of quantitative digital PCR experiments. Clinical Chemistry, 59(6):892-902, June 2013. [p127, 140]

J. F. Huggett, S. Cowen, and C. A. Foy. Considerations for digital PCR as an accurate molecular diagnostic tool. Clinical Chemistry, 61(1):79-88, Oct. 2015a. [p140]

J. F. Huggett, J. O'Grady, and S. Bustin. qPCR, dPCR, NGS - A journey. Biomolecular Detection and Quantification, 3:A1-A5, Mar. 2015b. [p127]

M. Jahn, J. Seifert, M. von Bergen, A. Schmid, B. Bühler, and S. Müller. Subpopulation-proteomics in prokaryotic populations. Current Opinion in Biotechnology, 24(1):79-87, Feb. 2013. [p141]

M. Jahn, C. Vorpahl, D. Türkowsky, M. Lindmeyer, B. Bühler, H. Harms, and S. Müller. Accurate determination of pasmid copy number of flow-sorted cells using droplet digital PCR. Analytical Chemistry, 86(12):5969-5976, June 2014. [p141]

D. A. Khodakov and A. V. Ellis. Recent developments in nucleic acid identification using solid-phase enzymatic assays. Microchimica Acta, 181(13-14):1633-1646, 2014. [p127]

J. D. Kloke and J. W. McKean. Rfit: Rank-based estimation for linear models. The R Journal, 4(2):57-64, Dec. 2012. [p139]

M. Kohl. SLqPCR: Functions for Analysis of Real-Time Quantitative PCR Data at SIRS-Lab GmbH. SIRS-Lab $\mathrm{GmbH}$, Jena, 2007. URL www. sirs-lab. com. [p128]

M. Kuhn. CRAN task view: Reproducible research, 2015. URL http://CRAN. R-project. org/view= ReproducibleResearch. Version 2015-06-18. [p129] 
T. J. Leeper. Archiving reproducible research and dataverse with R. The R Journal, 6(1):151-158, 2014. [p129]

S. Lefever, J. Hellemans, F. Pattyn, D. R. Przybylski, C. Taylor, R. Geurts, A. Untergasser, J. Vandesompele, and RDML Consortium. RDML: Structured language and reporting guidelines for real-time quantitative PCR data. Nucleic Acids Research, 37(7):2065-2069, Apr. 2009. [p128, 135]

Z. Liu and S. Pounds. An R package that automatically collects and archives details for reproducible computing. BMC Bioinformatics, 15(1):138, May 2014. [p129]

T. Luo, L. Jiang, W. Sun, G. Fu, J. Mei, and Q. Gao. Multiplex real-time PCR melting curve assay to detect drug-resistant mutations of mycobacterium tuberculosis. Journal of Clinical Microbiology, 49 (9):3132-3138, Sept. 2011. [p133]

N. Majumdar, T. Wessel, and J. Marks. Digital PCR modeling for maximal sensitivity, dynamic range and measurement precision. PLoS ONE, 10(3):e0118833, Mar. 2015. [p140]

J. Mar. qperNorm: Data-Driven Normalization Strategies for High-Throughput qPCR Data, 2009. URL http://bioconductor. org. R package version 1.26.0. [p128]

M. V. Matz. MCMC.qpcr: Bayesian Analysis of qRT-PCR Data, 2015. URL http: //CRAN. R-project.org/ package=MCMC. qper. R package version 1.2. [p128]

M. N. McCall1, H. R. McMurray, H. Land, and A. Almudevar. On non-detects in qPCR data. Bioinformatics, 30(16):2310-2316, Aug. 2014. [p128]

B. D. McCullough and D. A. Heiser. On the accuracy of statistical procedures in Microsoft Excel 2007. Computational Statistics \& Data Analysis, 52(10):4570-4578, June 2008. [p128]

P. Michna. RNetCDF: Interface to NetCDF Datasets, 2015. URL http://CRAN. R-project. org/package= RNetCDF. R package version 1.7-3; with contributions from Milton Woods. [p129]

P. Michna and M. Woods. RNetCDF - A package for reading and writing NetCDF datasets. The $R$ Journal, 5(2):29-37, Dec. 2013. [p129]

C. A. Milbury, Q. Zhong, J. Lin, M. Williams, J. Olson, D. R. Link, and B. Hutchison. Determining lower limits of detection of digital PCR assays for cancer-related gene mutations. Biomolecular Detection and Quantification, 1(1):8-22, Sept. 2014. [p127, 140]

A. A. Morley. Digital PCR: A brief history. Biomolecular Detection and Quantification, 1(1):1-2, Sept. 2014. [p127]

P. Murrell. It's not what you draw, it's what you don't draw. The R Journal, 4(2):13-18, Dec. 2012. [p128, 129]

P. Murrell. The gridGraphics package. The R Journal, 7(1):152-163, June 2015. [p128]

J. D. Neve and J. Meys. unifiedWMWqPCR: Unified Wilcoxon-Mann-Whitney Test for qPCR Data, 2014. URL http: //bioconductor. org. R package version 1.4.0. [p128]

J. D. Neve, J. Meys, J.-P. Ottoy, L. Clement, and O. Thas. unifiedWMWqPCR: The unified WilcoxonMann-Whitney test for analyzing RT-qPCR data in R. Bioinformatics, 30(17):2494-2495, 2014. [p128]

G. J. Nixon, H. F. Svenstrup, C. E. Donald, C. Carder, J. M. Stephenson, S. Morris-Jones, J. F. Huggett, and C. A. Foy. A novel approach for evaluating the performance of real time quantitative loopmediated isothermal amplification-based methods. Biomolecular Detection and Quantification, 2:4-10, Dec. 2014. [p127]

D. Nolan and D. L. Temple. XML and Web Technologies for Data Sciences with R. Springer-Verlag, New York, 2014. [p146]

J. Oh. Automatic conversion of tables to LongForm dataframes. The R Journal, 6(2):16-26, 2014. [p129]

A. Ohri. R for Cloud Computing - An Approach for Data Scientists. Springer-Verlag, New York, 2014. [p146]

J. Ooms. Directions for improved dependency versioning in R. The R Journal, 5(1):197-207, June 2013. [p129]

S. Pabinger, S. Rödiger, A. Kriegner, K. Vierlinger, and A. Weinhäusel. A survey of tools for the analysis of quantitative PCR (qPCR) data. Biomolecular Detection and Quantification, 1, 2014. [p127, 128, 140] 
Y. Pan, X. Yan, and J. Li. qPCR.CT: qPCR Data Analysis and Plot Package, 2012. URL http://CRAN. Rproject. org / package=qPCR. CT. R package version 1.1. [p128]

S. L. Pape. EasyqpcR: EasyqpcR for Easy Analysis of Real-Time PCR Data at IRTOMIT-INSERM U1082. IRTOMIT-INSERM U1082, 2012. URL http: //irtomit. labo. univ-poitiers. fr/. [p128]

J. R. Perkins, J. M. Dawes, C. Orengo, S. B. McMahon, D. L. Bennett, and M. Kohl. ReadqPCR and NormqPCR: $\mathrm{R}$ packages for the reading, quality checking and normalisation of RT-qPCR quantification cycle (Cq) data. BMC Genomics, 13(296), 2012. [p128]

R Core Team. R Data Import/Export. R Foundation for Statistical Computing, Vienna, Austria, 2015. URL http://www.R-project.org/. [p129]

C. Ritz and A.-N. Spiess. qpcR: an R package for sigmoidal model selection in quantitative real-time polymerase chain reaction analysis. Bioinformatics, 24(13):1549-1551, July 2008. PMID: 18482995. [p130]

S. Rödiger, T. Friedrichsmeier, P. Kapat, and M. Michalke. RKWard: A comprehensive graphical user interface and integrated development environment for statistical analysis with R. Journal of Statistical Software, 49(9):1-34, 2012. URL http://www. jstatsoft.org/v49/i09. [p129, 146]

S. Rödiger, A. Böhm, and I. Schimke. Surface melting curve analysis with R. The R Journal, 5(2):37-53, Dec. 2013a. [p128, 130, 133, 136, 138]

S. Rödiger, P. Schierack, A. Böhm, J. Nitschke, I. Berger, U. Frömmel, C. Schmidt, M. Ruhland, I. Schimke, D. Roggenbuck, W. Lehmann, and C. Schröder. A highly versatile microscope imaging technology platform for the multiplex real-time detection of biomolecules and autoimmune antibodies. Advances in Biochemical Engineering/Biotechnology, 133:35-74, 2013b. [p127, 128, 138]

S. Rödiger, C. Liebsch, C. Schmidt, W. Lehmann, U. Resch-Genger, U. Schedler, and P. Schierack. Nucleic acid detection based on the use of microbeads: A review. Microchimica Acta, 181(11-12): 1151-1168, 2014. [p127, 138]

S. Rödiger, M. Burdukiewicz, and P. Schierack. chipPCR: an R package to pre-process raw data of amplification curves. Bioinformatics, in press. doi: 10.1093/bioinformatics/btv205. [p130, 131, 139]

S. Roediger. MBmca: Nucleic Acid Melting Curve Analysis on Microbead Surfaces with R, 2015. URL http: //CRAN. R-project. org/package=MBmca. R package version 0.0.3-5. [p128]

S. Roediger and M. Burdukiewicz. chipPCR: Toolkit of Helper Functions to Pre-Process Amplification Data, 2014. URL http://CRAN. R-project.org/package=chipPCR. R package version 0.0.8-4. [p128]

RStudio Team. RStudio: Integrated Development Environment for R. RStudio, Inc., Boston, MA, 2012. URL http://www.rstudio.com/. [p129,160]

J. M. Ruijter, M. W. Pfaffl, S. Zhao, A. N. Spiess, G. Boggy, J. Blom, R. G. Rutledge, D. Sisti, A. Lievens, K. De Preter, S. Derveaux, J. Hellemans, and J. Vandesompele. Evaluation of qPCR curve analysis methods for reliable biomarker discovery: bias, resolution, precision, and implications. Methods, 59 (1):32-46, Jan. 2013. [p127, 128, 130, 133]

J. M. Ruijter, P. Lorenz, J. M. Tuomi, M. Hecker, and M. J. B. v. d. Hoff. Fluorescent-increase kinetics of different fluorescent reporters used for qPCR depend on monitoring chemistry, targeted sequence, type of DNA input and PCR efficiency. Microchimica Acta, 181(13-14):1689-1696, Oct. 2014. [p127]

D. A. Selck, M. A. Karymov, B. Sun, and R. F. Ismagilov. Increased robustness of single-molecule counting with microfluidics, digital isothermal amplification, and a mobile phone versus real-time kinetic measurements. Analytical Chemistry, 85(22):11129-11136, Nov. 2013. [p127]

A.-N. Spiess. qpcR: Modelling and Analysis of Real-Time PCR Data, 2014. URL http://CRAN. R-project. org/package=qpcR. R package version 1.4-0. [p128]

A.-N. Spiess, C. Deutschmann, M. Burdukiewicz, R. Himmelreich, K. Klat, P. Schierack, and S. Rödiger. Impact of smoothing on parameter estimation in quantitative DNA amplification. Clinical Chemistry, 61(2):379-388, 2015. [p128, 138]

D. Svec, A. Tichopad, V. Novosadova, M. W. Pfaffl, and M. Kubista. How good is a PCR efficiency estimate: Recommendations for precise and robust qPCR efficiency assessments. Biomolecular Detection and Quantification, 3:9-16, Mar. 2015. [p130] 
D. A. Swan. DivMelt: HRM Diversity Assay Analysis Tool, 2013. URL http: //CRAN. R-project.org/ package=DivMelt. R package version 1.0.3; with contributions from Craig A Magaret and Matthew M Cousins. [p128]

P. M. Valero-Mora and R. Ledesma. Graphical user interfaces for R. Journal of Statistical Software, 49(1): 1-8, June 2012. URL http: //www. jstatsoft. org/v49/i01. [p129]

M. van der Loo. settings: Software Option Settings Manager for R, 2015. URL http: //CRAN. R-project. org/package=settings. R package version 0.2.2. [p130]

E. Viturro, C. Altenhofer, B. Zölch, A. Burgmaier, I. Riedmaier, and M. W. Pfaffl. Microfluidic highthroughput reverse-transcription quantitative PCR analysis of liver gene expression in lactating animals. Microchimica Acta, 181(13-14):1725-1732, Oct. 2014. [p127]

W. Wang and G. Shan. ExactCIdiff: An R package for computing exact confidence intervals for the difference of two proportions. The R Journal, 5(2):62-71, Dec. 2013. [p144]

J. Wu, R. Kodzius, W. Cao, and W. Wen. Extraction, amplification and detection of DNA in microfluidic chip-based assays. Microchimica Acta, 181(13-14):1611-1631, Oct. 2014. [p127]

M. Zeller, W.-C. L. A. Guazzelli, and G. Williams. PMML: An open standard for sharing models. The R Journal, 1(1):60-65, June 2009. [p129]

J. D. Zhang, R. Biczok, and M. Ruschhaupt. ddCt: The ddCt Algorithm for the Analysis of Quantitative Real-Time PCR (qRT-PCR), 2015. URL http: //bioconductor. org. R package version 1.22.0. [p128]

Stefan Rödiger (corresponding author)

Faculty of Natural Sciences

Brandenburg University of Technology Cottbus-Senftenberg

Senftenberg

Germany

Stefan.Roediger@b-tu.de

Michat Burdukiewicz

University of Wroclaw

Faculty of Biotechnoloy

Department of Genomics

Wroclaw

Poland

michalburdukiewicz@gmail.com

Konstantin Blagodatskikh

All-Russia Research Institute of Agricultural Biotechnology

Center for collective use 'Biotechnology'

Moscow

Russia

k.blag@yandex.ru

Michael Jahn

Helmholtz Centre for Environmental Research - UFZ

Flow cytometry group / Environmental microbiology

Leipzig

Germany

michael.jahn@ufz.de

Peter Schierack

Faculty of Natural Sciences

Brandenburg University of Technology Cottbus-Senftenberg

Senftenberg

Germany

Peter.Schierackehs-lausitz.de 